\title{
Upper and lower bound solutions for lateral-torsional buckling of doubly symmetric members
}

\author{
by Arash Sahraei ${ }^{(1)}$ and Magdi Mohareb ${ }^{(1), *}$
}

(1) Department of Civil Engineering, University of Ottawa, Ottawa, ON, Canada K1N 6N5

\begin{abstract}
A family of three finite elements is developed for the lateral-torsional buckling analysis of thinwalled members with doubly symmetric cross-sections. The elements are based on a recently derived variational principle which incorporates shear deformation effects in conjunction with a special interpolation scheme ensuring $\mathrm{C} 1$ continuity. One of the elements is developed such that it consistently converges from above while another element is intended to consistently converge from below. The third element exhibits fast convergence characteristics compared to other shear deformable elements but cannot be guaranteed to provide either an upper or a lower bound solution. The formulation can incorporate any set of linear multi-point kinematic constraints. The validity of the solution is established through comparisons with other well-established numerical solutions. The elements are then used to solve practical problems involving simply supported beams, cantilevers and continuous beams under a variety of loading conditions including concentrated loads, linear bending moments and uniformly distributed loads. The effect of lateral and torsional restraints and the location of lateral restraint along the section height on lateraltorsional buckling capacity of beams are also examined through examples.
\end{abstract}

Keywords: Upper and lower bounds, computational efficiency, finite element, lateral-torsional buckling, lateral and torsional restraints, doubly symmetric sections, thin-walled members.

This article is to be cited as: Sahraei, A, Mohareb, M (2016), Upper and lower bound solutions for lateral-torsional buckling of doubly symmetric members, Thin-Walled Structures, 180-196, ISSN 0263-8231.

A copy-edited of this article can be obtained at: https://doi.org/10.1016/j.tws.2016.01.015

\footnotetext{
*Corresponding Author: Email: mmohareb@uottawa.ca
} 


\section{Motivation}

In a recent study, $\mathrm{Wu}$ and Mohareb [1, 2] developed a shear deformable theory and finite element formulation for lateral-torsional buckling of doubly-symmetric cross-sections. The element developed was based on linear interpolation of the displacement fields, leading to a $\mathrm{C} 0$ continuous element, and was shown to a) converge from above, in a manner similar to conventional finite element formulations, and b) to exhibit particularly slow-convergence characteristics as hundreds of degrees of freedom were needed to model simple problems. Starting with the same variational principle, the present study develops an elaborate interpolation scheme leading to $\mathrm{C} 1$ continuity and resulting in a family of finite elements for the lateraltorsional buckling analysis of members with superior characteristics; (1) It considerably accelerates the convergence characteristics of the solution, and (2) In one of the resulting elements, discretization errors are shown to lead to lower bound estimates of the buckling capacity, a desirable feature from a design viewpoint. In the second element, they were shown to lead to an upper bound estimate while the third element exhibits the fastest convergence characteristics. The new solution is subsequently used to investigate the effect of lateral and/or torsional restraints and the effect of lateral bracing location along the web height on the lateraltorsional buckling capacity of simple and multi-span beams.

\section{Literature review}

Numerous studies have investigated the elastic lateral-torsional buckling (LTB) resistance of doubly-symmetric I-beams. Using the Rayleigh-Ritz method, Salvadori [3] developed the LTB solution of simply supported and continuous beams subject to a combination of axial and unequal end moments. Based on the finite difference technique, Poley [4] solved the governing buckling differential equations for cantilever beams under uniformly distributed load. Using a successive-approximation technique for solving differential equations, Austin et al. [5] developed the critical LTB solutions for beams with full torsional end restraints and partial rotational end restraints about the weak axis subjected to uniformly distributed loads and midspan point loads. Load locations relative to the section centroid were also considered. Based on a numerical integration technique, Hartmann [6] evaluated the effect and partial lateral, torsional, 
and weak axis bending constraints on the LTB capacity of beams subjected to point loads, with interior supports for simply supported and continuous two-span and three-span beams. Krajcinovic [7] and Barsoum and Gallagher [8] developed a finite element for buckling analysis based on the Vlasov thin-walled beam theory. Powel and Klingner [9] developed a thin-walled beam finite element to obtain the LTB capacity of simply supported and continuous beams subject to general loading. Their solution was applicable to doubly symmetric and monosymmetric cross-sections. The effect of load position relative to shear center and that of lateral and torsional restraints were incorporated into the solution. Using the beam element developed by Gallagher and Padlog [10], Nethercot and Rockey [11] investigated the lateral stability of simply supported beams with discrete lateral restraints, discrete torsional restraints, and both lateral and torsional restraints, subject to uniform moments. Also, Based on the element, Nethercot [12] examined the effect of load type and lateral, torsional, and warping restraints on LTB of cantilevers and proposed expressions for the effective length of cantilevers governed by LTB. Using the same element, Nethercot [13] studied the effect of load type on LTB of simply supported beams braced laterally or torsionally under uniform moments, mid-span point load, and uniformly distributed load. Based on the finite integral method, Kitipornchai and Richter [14] studied the LTB of simply supported beams with discrete rigid intermediate translational and rotational restraints and subjected to concentrated load, end moments and uniformly distributed load. Kitipornchai et al. [15] extended their work to investigate the effect of intermediate translational and rotational discrete restraints on LTB capacity of cantilevers under uniformly distributed and concentrated loads. Based on the direct variational approach, Assadi and Roeder [16] studied the LTB of cantilevers with continuous rigid and elastic lateral restraints. Their study investigated the effects of load height, height of lateral restraint and stiffness. Based on a closed-form solution, Tong and Chen [17] investigated the LTB capacity of simply supported beams with symmetrical or mono-symmetrical I-sections, either restrained laterally or torsionally at mid-span, subjected to uniform bending moments. Wang and Nethercot [18] developed a thin-walled beam element for conducting a three-dimensional ultimate-strength analysis to assess bracing requirements for laterally unrestrained beams. They investigated simply supported I-beams with a single, three, or five equally spaced discrete torsional restraints subjected to central transverse concentrated load applied to the upper flange. Attard [19] 
developed solutions for estimating LTB capacity of beams with mono-symmetric and doubly symmetric sections and general boundary conditions. Albert et al. [20] developed a finite element model consisting of four-node plate elements for the web and two-node line elements for the flanges. This model predicts the LTB resistance of beams under various loading and boundary conditions while capturing distortional effects. Using this finite element model, Essa and Kennedy [21] developed effective length factors for built-in cantilevers under top and bottom flange lateral restraints and load positions relative to the shear center. Using the same element, they also developed a design approach for cantilever-suspended-span constructions [22]. Wang et al. [23] used the Rayleigh-Ritz method to determine the optimal locations for rigid lateral and torsional intermediate restraints to maximize the elastic LTB capacity of I-beams. Using the elastic buckling finite element program, BASP (Buckling Analysis of Stiffened Plates) developed by Akay et al. [24] and Choo [25], Yura [26] developed rules for bracing requirements based on the loading configuration, load height relative the shear center, location of restraint and cross-section distortion. Based on the Babnov-Galerkin method and the two-node beam finite element, Lim et al. [27] evaluated the effects of moment gradient and end warping, lateral, and torsional restraints on LTB capacity of beams. Park et al. [28] examined the effect of continuous top flange bracing on LTB capacity of simply supported and multi-span beams subject to uniformly distributed and concentrated loads based on the four-node plate element within the finite element program MSC/NASTRAN. Using shell analysis, Ozdemir and Topkaya [29] examined LTB capacity of overhanging monorails under various loading and boundary conditions. Based on a shell element solution and the finite difference method, Serna et al. [30] proposed a new moment gradient factor for simply supported beams restrained torsionally at both ends and subject to general loads applied at the shear center. Using the Rayleigh-Ritz method, Andrade et al. [31] extended the application of the Eurocode 3 [32] three-factor method for LTB to cantilevers with doubly and mono-symmetric sections subject to uniformly distributed and concentrated transverse tip loads. Their solution incorporated the effect of load height relative to the shear center. Larue et al. [33] developed a code based on a mathematical software, called MGv3, for determining the LTB moment of I-beams with rigid continuous lateral restraint applied at the tension flange. The same problem was solved by Khelil and Larue [34] who adopted the Galerkin method. Using a finite element program, FTBER, an extension of 
the commercial finite element program, PRFELB, Trahair [35] developed a design approach for obtaining lateral buckling resistance of monorail beams, cantilevers and overhangs loaded at the bottom flange and laterally and transversely supported at the top flange. Based on the RayleighRitz approach, Nguyen et al. [36] developed LTB solutions for I-girders subject to linear bending moments with equidistant discrete torsional restraints and quantified the torsional stiffness requirements for the restraints to maximize the LTB capacity.

A common feature about the previous studies is the fact that they neglect shear deformation. Shear deformable buckling solutions were developed relatively recently. Using the stationary complementary energy variational principle, Erkmen and Mohareb [37, 38] developed a shear deformable element to determine the LTB capacity of open thin-walled members. Erkmen et al. [39] investigated the conditions under which the elastic torsional buckling of columns is guaranteed to converge from below. Based on a hyper-elastic constitutive model, Attard and Kim [40] developed a shear deformable element to determine LTB solutions of simply supported beams subject to uniform bending moment. Another shear deformable element was developed by $\mathrm{Wu}$ and Mohareb $[1,2]$ based on the principle of stationary potential energy. Erkmen and Attard [41] and Erkmen [42] also developed shear deformable elements to predict LTB capacity of thinwalled members. Examples on shear deformable elements in composite beams include the work of Kim and Lee [43] and Kim and Choi [44]. Within the above context, the present solution provides a shear deformable solution.

In the majority of the above studies, discretization errors are observed to lead to upper bound predictions of the critical loads. Exceptions to that trend are observed in [37, 38], [39], [42], [45] and [46] which provide lower bound predictions of the critical loads in some of problems investigated. However, none of the above studies reached an unconditional lower bound buckling load prediction. In this context, one of the finite elements developed under the present study is shown to consistently provide a lower bound buckling prediction.

\section{Assumptions}

The variational principle [2] is based on the following assumptions:

1. Beam cross-sections are open and doubly-symmetric, 
2. Regarding shear/bending action, the cross-section is assumed to remain rigid in its own plane during deformation but does not remain perpendicular to the neutral axis after deformation in line with the Timoshenko theory [1]. The hypothesis is further generalized for torsion/warping action,

3. Strains are assumed small but rotations are assumed to be moderate. Rotation effects are thus included in the formulation by retaining the non-linear strain components,

4. The member buckles in an inextensional mode [47] which means that throughout buckling, the centroidal longitudinal strain and curvature in yz-plane remain zero. This signifies that the member is assumed to buckle under constant axial load and bending moments,

5. The material is assumed to be linearly elastic and obeys the Hooke's law, and

6. Pre-buckling deformation and distortional effects are neglected.

\section{Problem description, convention and notation}

A right-handed Cartesian coordinate system is adopted in the present study. The z-axis is oriented along the axial direction while $\mathrm{x}$-axis and $\mathrm{y}$-axis are parallel to major and minor principal axes, respectively. The origin coincides with the cross-section centroid $C\left(x_{c}=0, y_{c}=0\right)$, sectorial origin $S_{0}$, Pole $A_{p}$ and shear center $S C$ to forms an orthogonal coordinate system. The member is assumed to be subjected to a uniformly distributed transverse load $q_{y}$ applied at a distance $y_{q y}(z)$ from the shear center and a uniformly distributed axial load $q_{z}$ acting at distance $y_{q z}(z)$ from the origin. Under such external loads, the member deforms from configuration 1 to 2 as shown in Fig. 1 and undergoes displacements $v_{p}(z), w_{p}(z)$ and rotation $\theta_{x p}(z)$. As a convention, subscript $p$ represents pre-buckling displacement, strain and stress fields. The applied loads are assumed to increase by a factor $\lambda$ and attain the values $\lambda q_{y}$ and $\lambda q_{z}$ at the onset of buckling (Configuration 3). Under the load increase, it is assumed that pre-buckling deformations linearly increase to $\lambda v_{p}(z), \lambda w_{p}(z)$ and $\lambda \theta_{p}(z)$. The section then undergoes LTB (Configuration 4) manifested by lateral displacement $u_{b}$, weak-axis rotation $\theta_{y b}$, 
angle of twist $\theta_{z b}$ and warping deformation $\psi_{b}$. Again, as a matter of convention, subscript $b$ denotes field displacements, strains, or stresses, occurring during the buckling stage (i.e., in going from configuration 3 to 4 ).

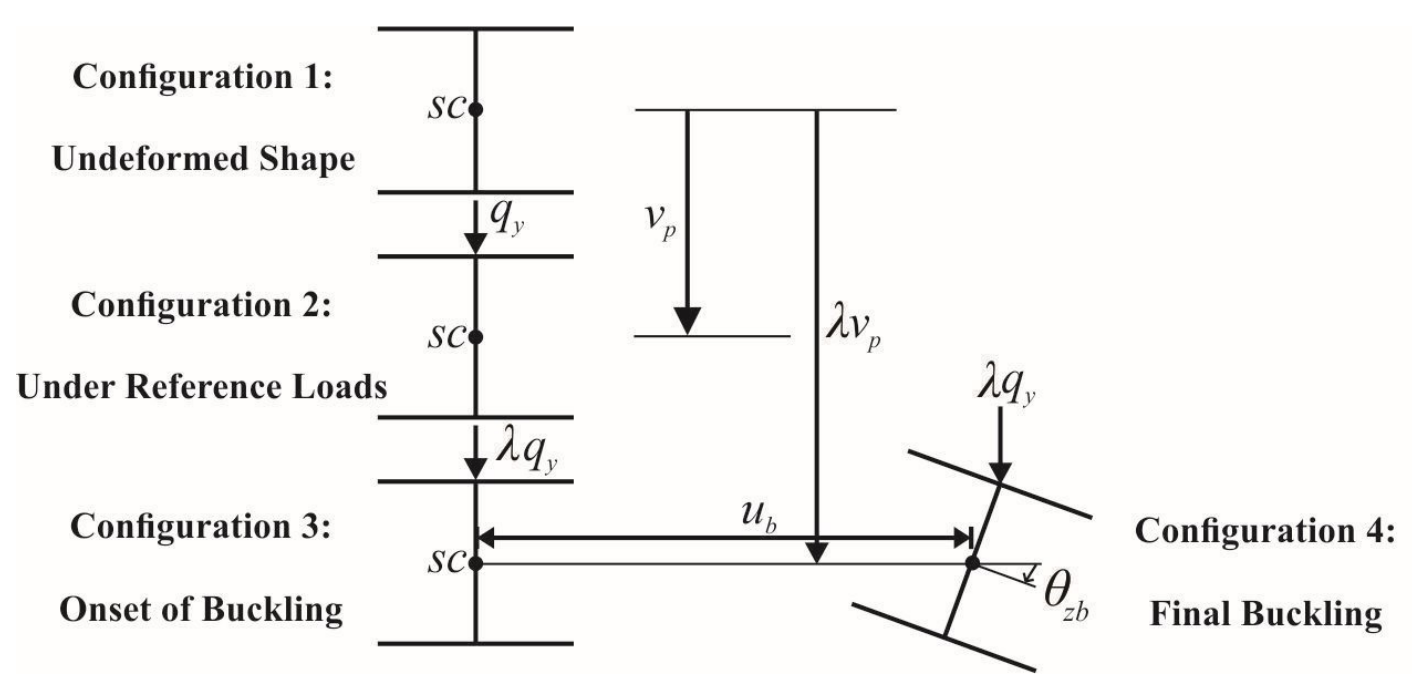

Fig. 1. Different stages of deformation

\section{Variational principle}

When the member is at the onset of buckling (Configuration 3 in Fig. 1), the first variation of total potential energy $\delta \pi$ has to vanish to meet the equilibrium condition and also the variation of the second variation of total potential energy $\delta^{2} \pi$ has to vanish to meet condition of neutral stability, i.e., $\delta\left[(1 / 2) \delta^{2} \pi\right]=\delta\left[(1 / 2) \delta^{2}(U+V)\right]=0$, in which, $U$ is the internal strain energy and $V$ is the load potential energy gained by externally applied loads. For a doubly-symmetric cross-section and using orthogonal coordinates, the condition of neutral stability leads to [1]

$\delta\left(\frac{1}{2} \delta^{2} \pi\right)=\delta\left[\frac{1}{2} \delta^{2}\left(U_{b}+U_{s v}+U_{s}+V_{N}+V_{M}+V_{V}+V_{q y}+V_{q z}\right)\right]=0$

in which, $U_{b}, U_{s v}$ and $U_{s}$ are, respectively, the internal strain energy due to normal stresses, Saint-Venant shear stresses, and other shearing stresses induced by bending and warping. The terms $V_{N}, V_{M}, V_{V}, V_{q y}$ and $V_{q z}$ represent the destabilizing effects due to normal forces, bending 
moments, shear forces, transverse load position effect, and longitudinal load position effect, respectively. For orthogonal coordinates, the second variations of the above energy terms take the form $[1,2]$

$$
\begin{aligned}
\delta^{2} U_{b}= & \int_{0}^{L}\left[E I_{y y}\left(\theta_{y b}^{\prime}\right)^{2}+E I_{\omega \omega}\left(\psi_{b}^{\prime}\right)^{2}\right] d z \\
\delta^{2} U_{s v}= & \int_{0}^{L} G J\left(\theta_{z b}^{\prime}\right)^{2} d z \\
\delta^{2} U_{s}= & G \int_{0}^{L}\left[\left(D_{x x} u_{b}^{\prime}-D_{x x} \theta_{y b}\right) u_{b}^{\prime}+\left(-D_{x x} u_{b}^{\prime}+D_{x x} \theta_{y b}\right) \theta_{y b}+\left(D_{h h} \theta_{z b}^{\prime}+D_{h h} \psi_{b}\right) \theta_{z b}^{\prime}\right. \\
& \left.+\left(D_{h h} \theta_{z b}^{\prime}+D_{h h} \psi_{b}\right) \psi_{b}\right] d z \\
\delta^{2} V_{N}= & -\lambda \int_{0}^{L}\left(\frac{N_{p}(z)}{A}\right)\left[-A u_{b}^{\prime 2}-\left(I_{x x}+I_{y y}\right) \theta_{z b}^{\prime 2}\right] d z \\
\delta^{2} V_{M}= & -\lambda \int_{0}^{L} M_{x p}(z)\left[-2\left(\theta_{y b}^{\prime} \theta_{z b}+\theta_{y b} \theta_{z b}^{\prime}\right)+2 u_{b}^{\prime} \theta_{z b}^{\prime}\right] d z \\
\delta^{2} V_{V}= & -\lambda \int_{0}^{L} V_{y p}(z)\left(-2 \theta_{y b} \theta_{z b}+2 u_{b}^{\prime} \theta_{z b}\right) d z \\
\delta^{2} V_{q y}= & -\lambda \int_{0}^{L}-q_{y} y_{q y}\left(\theta_{z b}\right)^{2} d z \\
\delta^{2} V_{q z}= & -\lambda \int_{0}^{L} 2 q_{z} y_{q z} \theta_{y b} \theta_{z b} d z
\end{aligned}
$$

in which, $E$ is Young's modulus, $A$ is the cross-sectional area, $G$ is the shear modulus, $J$ is the Saint-Venant torsional constant and the cross-sectional properties $I_{y y}=\int_{A} x^{2} d A$, $I_{\omega \omega}=\int_{A} \omega^{2} d A, \quad D_{h h}=\int_{A} h^{2} d A, \quad D_{x x}=\int_{A}(d x / d s)^{2} d A$ have been defined. Also, $\omega$ is the Vlasov warping function, $s$ is a tangential coordinate along the section mid-surface and $h$ is the distance perpendicular from the section shear center to the tangent to the section mid-line at the point of interest. In Eqs. (2a-h), the pre-buckling internal forces $N_{p}(z), M_{x p}(z), V_{y p}(z)$ denote the normal force, bending moments about the strong axis, and associated shear force, respectively. 


\section{Overview of the finite element formulation in $\mathrm{Wu}$ and Mohareb [2]}

The variational expressions in Eqs (2a-h) consist of the buckling displacement functions $\begin{array}{lllll}u_{b} & \theta_{y b} & \theta_{z b} & \psi_{b}\end{array}$ and their first derivatives with respect to coordinate $z$. Thus, each of the assumed four displacement functions needs to satisfy only $\mathrm{C} 0$ continuity. By taking two nodes per element and adopting a linear interpolation scheme between the two nodal values, the displacement fields $\left\langle u_{b}(z) \quad \theta_{y b}(z) \quad \theta_{z b}(z) \quad \psi_{b}(z)\right\rangle^{T}$ were related [2] to the nodal displacements through

$\left\langle u_{b}(z) \quad \theta_{y b}(z) \quad \theta_{z b}(z) \quad \psi_{b}(z)\right\rangle_{1 \times 4}=\left\langle H_{b}(z)\right\rangle_{1 \times 2}\left[\left\{\begin{array}{l}u_{1} \\ u_{2}\end{array}\right\}_{2 \times 1} \quad\left\{\begin{array}{l}\theta_{y 1} \\ \theta_{y 2}\end{array}\right\}_{2 \times 1} \quad\left\{\begin{array}{l}\theta_{z 1} \\ \theta_{z 2}\end{array}\right\}_{2 \times 1}\left\{\begin{array}{l}\psi_{1} \\ \psi_{2}\end{array}\right\}_{2 \times 1}\right]$

in which, $\left\langle H_{b}(z)\right\rangle_{1 \times 2}=\langle(1-z / L) \quad(z / L)\rangle$ is the vector of linear shape functions and $u_{1}, u_{2}, \theta_{y 1}, \ldots . \psi_{2}$ are the nodal displacement. In a similar manner, the pre-buckling stress resultants $N_{p}(z) V_{y p}(z) M_{x p}(z)$ are linearly interpolated between the internal forces $N_{1}, N_{2}, V_{1}, V_{2}, M_{1}, M_{2}$ at the nodes as obtained from the pre-buckling analysis, i.e.,

$$
\left\langle N_{p}(z) \quad V_{y p}(z) \quad M_{x p}(z)\right\rangle=\left\langle H_{b}(z)\right\rangle_{1 \times 2}\left[\left\{\begin{array}{l}
N_{1} \\
-N_{2}
\end{array}\right\}_{2 \times 1} \quad\left\{\begin{array}{l}
V_{1} \\
-V_{2}
\end{array}\right\}_{2 \times 1} \quad\left\{\begin{array}{l}
M_{1} \\
-M_{2}
\end{array}\right\}_{2 \times 1}\right]
$$

The resulting element, to be subsequently referred as the WM element, was successful in converging to the buckling solutions [2] only when a rather large number of elements were taken. Coarser meshes were observed to grossly overestimate buckling loads due to shear locking. Thus, the present formulation circumvents such problems by developing special shape functions featuring $\mathrm{C} 1$ continuity while preserving the minimal number degrees of freedom adopted in the WM element. 


\section{Conditions of neutral stability}

Eqs. (2a-h) are integrated by parts and common terms are grouped together. Since functions $\delta u_{b}, \delta \theta_{y b}, \delta \theta_{z b}$ and $\delta \psi_{b}$ are arbitrary, one can rewrite the conditions of neutral stability in a matrix form as

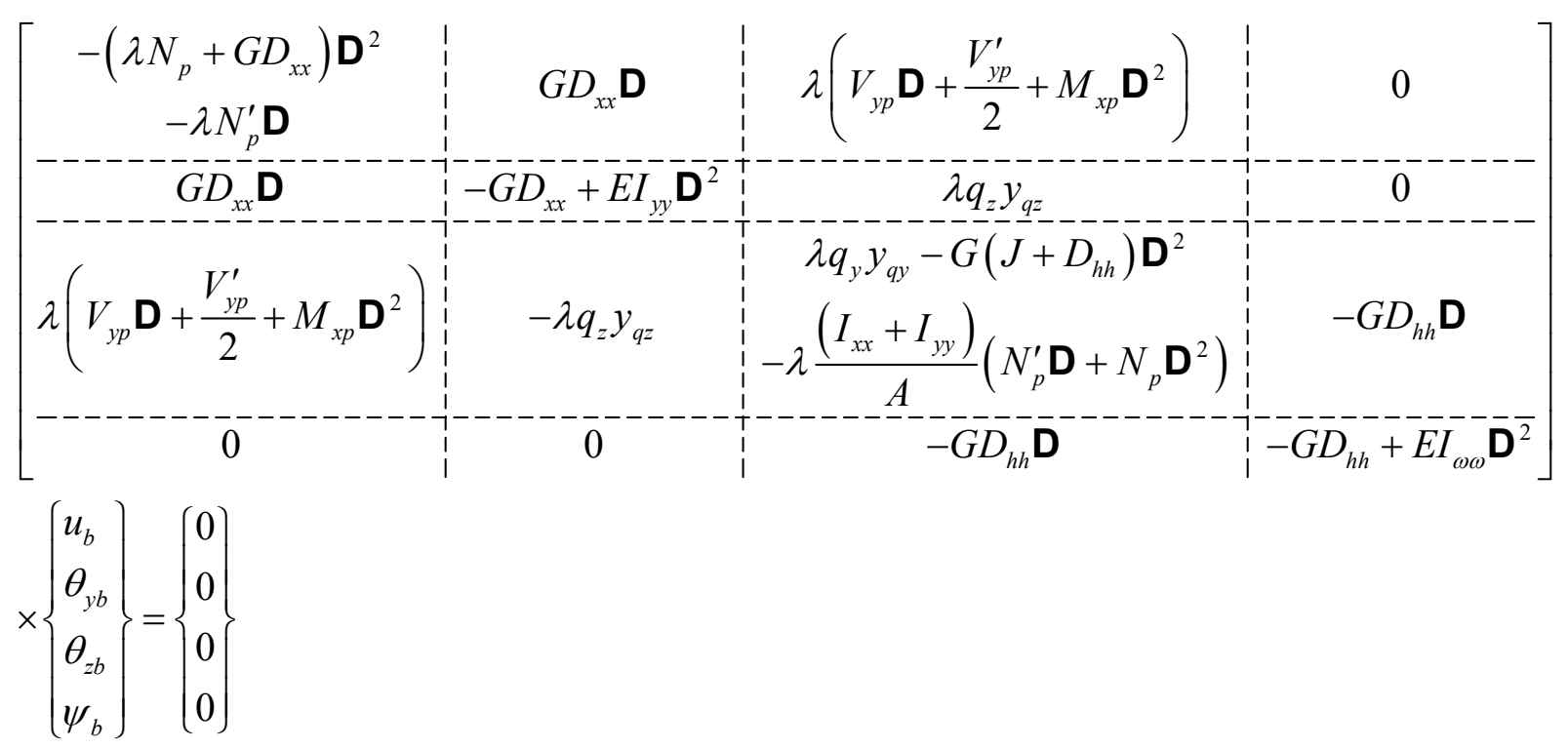

in which, $\mathbf{D}=\partial / \partial z, \mathbf{D}^{2}=\partial^{2} / \partial z^{2}$.

\section{Finite element formulation}

As discussed earlier, the element developed in [2] has a minimum number of degrees of freedom (8 DOFs for the buckling solution) but exhibited slow-convergence, thus needing hundreds of elements to model simple problems. In order to develop computationally more efficient solutions, the present study devises a set of approximation scheme of the pre-buckling internal forces (Section 8.1), followed by formulating shape functions with superior convergence characteristics (Section 8.2) while keeping the number of degrees of freedom per element to a minimum. These shape functions are then used to develop a family of finite elements with various superior characteristics. 


\subsection{Approximation of pre-buckling internal forces}

In general, the pre-buckling internal forces $N_{p}(z), V_{y p}(z)$ and $M_{x p}(z)$ are non-constant functions. Conventional solutions (e.g., [8], [2]) capture the exact distribution of internal forces [Fig. 2(a)], as predicted from the pre-buckling analysis, when formulating the geometric stiffness matrices arising from Eqs. (2d-h). In the present study, we deviate from this convention by subdividing the member(s) into a series of small elements such that the internal forces $N_{p}(z) \approx \bar{N}_{p}, V_{y p}(z) \approx \bar{V}_{y p}$ and $M_{x p}(z) \approx \bar{M}_{x p}$ can be considered constant within each element. The advantages of the proposed treatment are:

a) Unlike the case where $N_{p}(z), V_{y p}(z)$ and $M_{x p}(z)$ are non-constant, under the approximations $N_{p}(z) \approx \bar{N}_{p}, V_{y p}(z) \approx \bar{V}_{y p}$ and $M_{x p}(z) \approx \bar{M}_{x p}$, a closed-form solution for the equilibrium equation system [Eq. (5)] becomes attainable. This outcome is key in developing the superior shape functions proposed under the present study (Section 8.2).

b) It is clear that the proposed approximation would converge to the correct solution when enough elements are taken, and

c) Within each element, the magnitudes of constants $\bar{N}_{p}, \bar{V}_{y p}$ and $\bar{M}_{x p}$ can be taken to target either a fast-converging solution (Case 1), an upper bound solution (Case 2), or a lower bound solution (Case 3) as describe in the following section.

\section{Case 1: Fast-converging solution}

Under this case, the internal forces are taken equal to the mean value of the internal forces [Fig. 2(b)] within the element i.e.,

$$
\left[N_{p}(z), V_{y p}(z), M_{x p}(z)\right] \approx\left(\bar{N}_{p}, \bar{V}_{y p}, \bar{M}_{x p}\right)=\left[\left(N_{1}-N_{2}\right) / 2 \quad,\left(V_{1}-V_{2}\right) / 2, \quad\left(M_{1}-M_{2}\right) / 2\right]
$$

A similar approach was adopted in [48] for mono-symmetric sections. It is stressed however, that the solution in [48] is not applicable to doubly symmetric sections which are the focus of the present study. This is the case since, as will be discussed under Section 8.2, the closed-form 
solution for the neutral stability conditions for the present problem as given in Eq. (5) takes a different form from that reported in [48]. Thus, the direct application of the solution in [48] is found to lead to singularity problems when applied to doubly symmetric sections. Within this context, the present treatment circumvents these difficulties by treating the case of doubly symmetric separately. The finite element resulting from the approximation in Eq. (6a-c) will be shown to have excellent convergence characteristics compared to that in [2]. The element resulting from Case 1 will be referred to as the SM-M element.

\section{Case 2: Upper-bound solution}

An upper bound prediction of the buckling load is targeted by adopting internal force approximations which consistently underestimate the destabilizing effect as given by Eqs. (2d-h), leading to a larger buckling load multiplier $\lambda$ and hence over-predicting the critical loads. The following approximations satisfy this requirement

$$
\begin{aligned}
& N_{p}(z) \approx \bar{N}_{p}=s_{n}\left(N_{1}, N_{2}\right) \min \left(\left|N_{1}\right|,\left|N_{2}\right|\right) \\
& V_{y p}(z) \approx \bar{V}_{y p}=s_{n}\left(V_{1}, V_{2}\right) \min \left(\left|V_{1}\right|,\left|V_{2}\right|\right) \\
& M_{x p}(z) \approx \bar{M}_{x p}=s_{n}\left(M_{1}, M_{2}\right) \min \left(\left|M_{1}\right|,\left|M_{2}\right|\right)
\end{aligned}
$$

where, $s_{n}\left(a_{1}, a_{2}\right)$ is the sign of argument $\left(a_{1}\right.$ or $\left.a_{2}\right)$ with the smaller absolute value. Mathematically, it is given by

$$
s_{n}\left(a_{1}, a_{2}\right)= \begin{cases}+1 & \text { if }\left[\left(a_{1}>0\right) \text { and }\left(a_{2}>0\right)\right] \\ -1 & \text { if }\left[\left(a_{1}>0\right) \text { and }\left(a_{2}<0\right) \text { and }\left(\left|a_{1}\right|>\left|a_{2}\right|\right)\right] \\ +1 & \text { if }\left[\left(a_{1}>0\right) \text { and }\left(a_{2}<0\right) \text { and }\left(\left|a_{1}\right| \leq\left|a_{2}\right|\right)\right] \\ -1 & \text { if }\left[\left(a_{1}<0\right) \text { and }\left(a_{2}<0\right)\right] \\ +1 & \text { if }\left[\left(a_{1}<0\right) \text { and }\left(a_{2}>0\right) \text { and }\left(\left|a_{1}\right|>\left|a_{2}\right|\right)\right] \\ -1 & \text { if }\left[\left(a_{1}<0\right) \text { and }\left(a_{2}>0\right) \text { and }\left(\left|a_{1}\right| \leq\left|a_{2}\right|\right)\right] \\ 0 & \text { if }\left[\left(a_{1}=0\right) \text { or }\left(a_{2}=0\right)\right]\end{cases}
$$


It is clear that, as the number of elements are increased, Equations (7a-c) lead to $N_{p}(z) \rightarrow \bar{N}_{p}$, $V_{y p}(z) \rightarrow \bar{V}_{y p}, M_{x p}(z) \rightarrow \bar{M}_{x p}$ and in the limit, the approximate destabilizing term approaches that based on the exact distribution of the internal forces within the element, i.e., the solution is guaranteed to converge to the buckling load from above. The element based on approximations (7a-c) will be referred to as the SM-N element [Fig. 2(c)].

\section{Case 3: Lower-bound solution}

In a similar manner, a guaranteed lower bound prediction of the buckling load is targeted by adopting internal force approximations which consistently overestimating the magnitudes of the destabilizing terms as given by Eqs. (2d-h), thus leading to a smaller buckling load multiplier $\lambda$. Towards this goal, the following approximations are proposed

$$
\begin{aligned}
& N_{p}(z) \approx \bar{N}_{p}=s_{x}\left(N_{1}, N_{2}\right) \max \left(\left|N_{1}\right|,\left|N_{2}\right|\right) \\
& V_{y p}(z) \approx \bar{V}_{y p}=s_{x}\left(V_{1}, V_{2}\right) \max \left(\left|V_{1}\right|,\left|V_{2}\right|\right) \\
& M_{x p}(z) \approx \bar{M}_{x p}=s_{x}\left(M_{1}, M_{2}\right) \max \left(\left|M_{1}\right|,\left|M_{2}\right|\right)
\end{aligned}
$$

where $s_{n}\left(a_{1}, a_{2}\right)$ is the sign of argument ( $a_{1}$ or $\left.a_{2}\right)$ with the larger absolute value, i.e.,

$$
s_{x}\left(a_{1}, a_{2}\right)= \begin{cases}+1 & \text { if }\left[\left(a_{2} \geq-a_{1}\right) \operatorname{and}\left(a_{1}>0\right)\right] \\ -1 & \text { if }\left[\left(a_{2}<-a_{1}\right) \operatorname{and}\left(a_{1}>0\right)\right] \\ +1 & \text { if }\left[\left(a_{2}>-a_{1}\right) \operatorname{and}\left(a_{1}<0\right)\right] \\ -1 & \text { if }\left[\left(a_{2} \leq-a_{1}\right) \text { and }\left(a_{1}<0\right)\right] \\ \operatorname{sign}\left(a_{1}\right) & \text { if } a_{2}=0 \\ \operatorname{sign}\left(a_{2}\right) & \text { if } a_{1}=0 \\ 0 & \text { if }\left[\left(a_{1}=0\right) \text { and }\left(a_{2}=0\right)\right]\end{cases}
$$

The resulting element will be referred to as the SM-X element [Fig. 2(d)] and will be shown to consistently converge from below. 


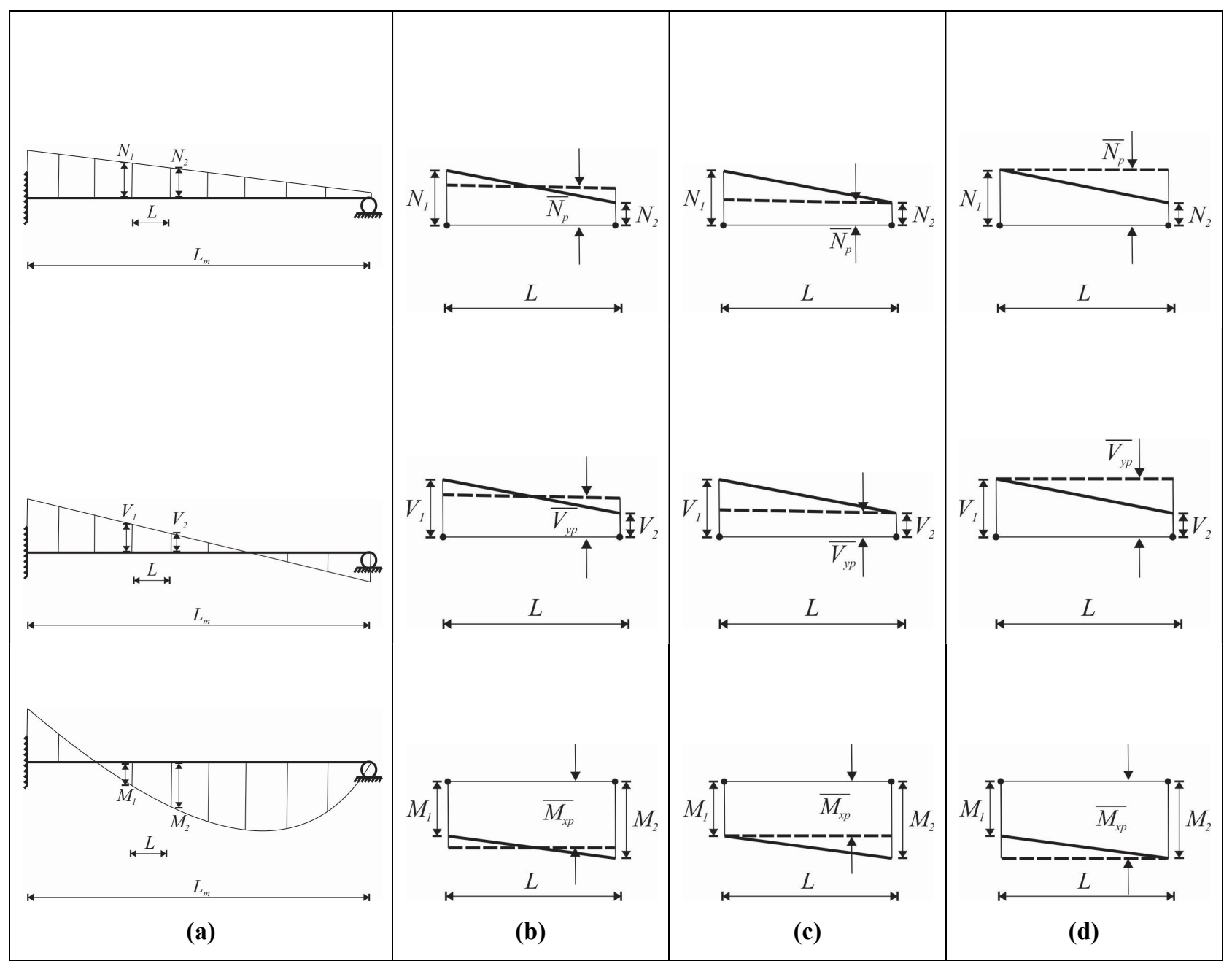

Fig. 2. (a) Pre-buckling internal forces and approximations for (b) SM-M element, (c) SM-N element and (d) SM-X element (all solid lines denote exact internal force diagrams and dashed lines denote internal force approximations)

Within an element length $L$, any of the approximations provided in Eqs. (6a-c), (7a-c) or (8a-c) leads to the following approximate expressions for the destabilizing energy terms in Eqs. (2d-f)

$$
\begin{aligned}
& \delta^{2} V_{N} \approx-\lambda \bar{N}_{p} \int_{0}^{L}\left(\frac{1}{A}\right)\left[-A u_{b}^{\prime 2}-\left(I_{x x}+I_{y y}\right) \theta_{z b}^{\prime 2}\right] d z \\
& \delta^{2} V_{M} \approx-\lambda \bar{M}_{x p} \int_{0}^{L}\left[-2\left(\theta_{y b}^{\prime} \theta_{z b}+\theta_{y b} \theta_{z b}^{\prime}\right)+2 u_{b}^{\prime} \theta_{z b}^{\prime}\right] d z \\
& \delta^{2} V_{V} \approx-\lambda \bar{V}_{y p} \int_{0}^{L}\left(-2 \theta_{y b} \theta_{z b}+2 u_{b}^{\prime} \theta_{z b}\right) d z
\end{aligned}
$$


It is clear that under all three approximations in Cases (1-3), as the number of elements is sufficiently large, the piecewise approximations of the destabilizing terms in Eqs. (9a-c) would approach that based on actual internal force distributions in Eqs. (2d-f), thus guaranteeing convergence to the critical loads.

\subsection{Formulating shape functions}

Since the interpolation scheme which will be presented in this section, uses shape functions that are developed based on closed-form solutions, the presence of the unknowns $\lambda N_{p}, \lambda V_{y p}, \lambda M_{x p}$ arising in Eq. (5), makes such a solution unattainable given that $\lambda$ is unknown a-priori. Thus, when formulating shape functions, the terms involving $\lambda N_{p}, \lambda V_{y p}, \lambda M_{x p}$ are assumed negligible. The validity of this hypothesis is numerically assessed and verified in Appendix A.

It is emphasized that this simplification will be used only for the purpose of developing shape functions. Once the shape functions are developed, they will be substituted into the stationary condition of the second variation of the total potential energy functional [Eq. (1)] while retaining the contributions $\delta^{2} V_{N}, \delta^{2} V_{M}, \delta^{2} V_{V}$ of destabilizing terms

By neglecting the destabilizing terms, the differential equations of neutral stability can be expressed as two sets of coupled differential equations [Eq. (A2)]

$$
\begin{aligned}
& {\left[\begin{array}{cc}
-G D_{x x} \mathbf{D}^{2} & G D_{x x} \mathbf{D} \\
G D_{x x} \mathbf{D} & -G D_{x x}+E I_{y y} \mathbf{D}^{2}
\end{array}\right]\left\{\begin{array}{l}
u_{b} \\
\theta_{y b}
\end{array}\right\}=\left\{\begin{array}{l}
0 \\
0
\end{array}\right\}} \\
& {\left[\begin{array}{cc}
-G J \mathbf{D}^{2}-G D_{h h} \mathbf{D}^{2} & -G D_{h h} \mathbf{D} \\
-G D_{h h} \mathbf{D} & -G D_{h h}+E I_{\omega \omega} \mathbf{D}^{2}
\end{array}\right]\left\{\begin{array}{l}
\theta_{z b} \\
\psi_{b}
\end{array}\right\}=\left\{\begin{array}{l}
0 \\
0
\end{array}\right\}}
\end{aligned}
$$

The solution is assumed to take the form of $\left\langle u_{b}, \theta_{y b}, \theta_{z b}, \psi_{b}\right\rangle^{T}=\left\langle A_{i}, B_{i}, C_{i}, D_{i}\right\rangle^{T} e^{m_{i} z}$. By substituting into Eqs. (10a-b) and expanding the determinant of coefficients for each set, one has

$$
\left[\begin{array}{c:c}
-G D_{x x} m^{2} & G D_{x x} m \\
\hdashline G D_{x x} m & -G D_{x x}+E I_{y y} m^{2}
\end{array}\right]\left\{\begin{array}{l}
A \\
B
\end{array}\right\}=\left\{\begin{array}{l}
0 \\
0
\end{array}\right\}, \quad\left[\begin{array}{cc}
-G J m^{2}-G D_{h h} m^{2} & -G D_{h h} m \\
\hdashline-G D_{h h} m & -G D_{h h}+E I_{\omega \omega} m^{2}
\end{array}\right]\left\{\begin{array}{l}
C \\
D
\end{array}\right\}=\left\{\begin{array}{l}
0 \\
0
\end{array}\right\}
$$


By setting the determinant of the matrix of coefficients of Eq. (11a) to zero, one obtains four zero roots, i.e., $m_{1}=m_{2}=m_{3}=m_{4}=0$. Also, by setting the determinant of the matrix of coefficients of Eq. (11b) to zero, one obtains

$m_{1}=m_{2}=0, \quad m_{3}=-m_{4}=\sqrt{\frac{G J D_{h h}}{E I_{\omega \omega}\left(D_{h h}+J\right)}}$

The corresponding solution can be shown to take the form

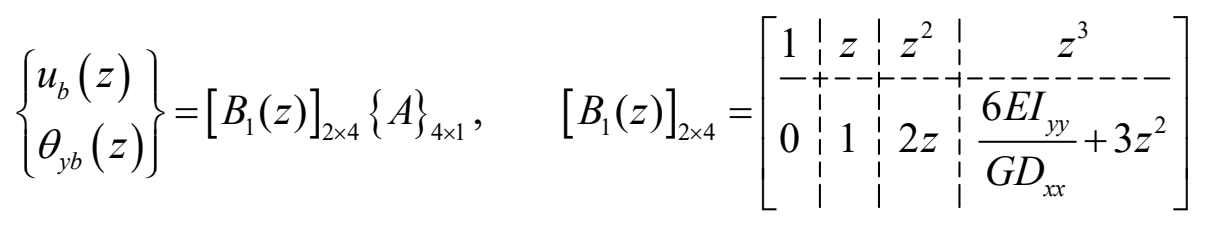

where, the vector of integration constants $\langle A\rangle^{T}=\left\langle\begin{array}{llll}A_{1} & A_{2} & A_{3} & A_{4}\end{array}\right\rangle$ has been defined. Also, one has

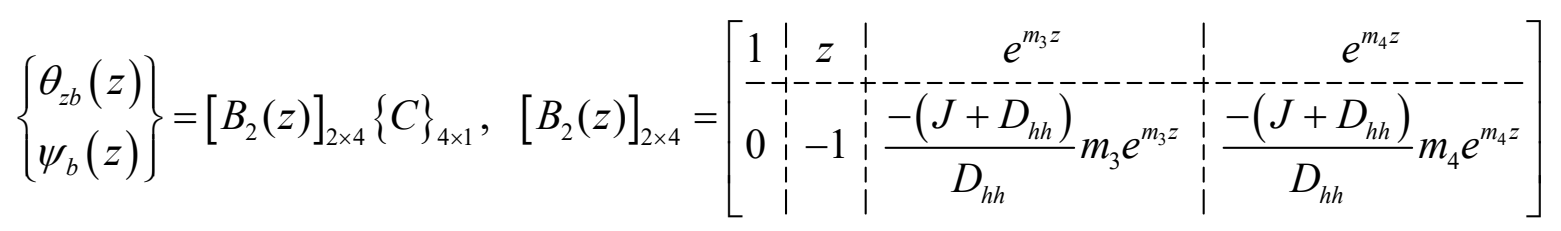

$(14 a-b)$

where, vector $\langle C\rangle^{T}=\left\langle\begin{array}{llll}C_{1} & C_{2} & C_{3} & C_{4}\end{array}\right\rangle$ consists of the integration constants. Equations (13a) and (14a) are consolidated to yield

$\{d(z)\}_{4 \times 1}=\left[\begin{array}{c:c}{\left[B_{1}(z)\right]} & {[0]} \\ \hdashline[0] & {\left[B_{2}(z)\right]}\end{array}\right]_{4 \times 8}\left\{C_{s t}\right\}_{8 \times 1}$

where, $\langle d(z)\rangle^{T}=\left\langle u_{b}(z) \quad \theta_{y b}(z) \quad \theta_{y b}(z) \quad \psi_{b}(z)\right\rangle^{T}$ consist of the displacement functions, and the vector of integration constants $\left\langle C_{s t}\right\rangle^{T}=\left\langle\langle A\rangle^{T} \mid\langle C\rangle^{T}\right\rangle$ has been defined. The nodal 
displacements $\left\langle\delta u_{N}\right\rangle^{T}=\left\langle\begin{array}{llllllll}\delta u_{b 1} & \delta \theta_{y b 1} & \delta \theta_{z b 1} & \delta \psi_{b 1} & \delta u_{b 2} & \delta \theta_{y b 2} & \delta \theta_{z b 2} & \delta \psi_{b 2}\end{array}\right\rangle^{T}$ can be related to the vector of integration constants $\left\{C_{s t}\right\}$ by setting $z=0$ and $z=L$ in Eq. (15) via

$$
\left\{\delta u_{N}\right\}_{8 \times 1}=[H]_{8 \times 8}\left\{C_{s t}\right\}_{8 \times 1}, \quad[H]_{8 \times 8}=\left[\begin{array}{l}
{[B(0)]_{4 \times 8}} \\
{[B(\bar{l})]_{4 \times 8}^{-}}
\end{array}\right]
$$

From Eq. (16a), by solving for the integration constants and substituting into Eq. (15), one obtains

$\{d(z)\}_{4 \times 1}=[L(z)]_{4 \times 8}\left\{\delta u_{N}\right\}_{8 \times 1}$

in which, $[L(z)]_{4 \times 8}=[B(z)]_{4 \times 8}[H]_{8 \times 8}^{-1}$ is a matrix of 32 shape functions. Shape functions $[L(z)]_{4 \times 8}$ ensure $\mathrm{C} 1$ continuity of the displacement fields while exactly satisfy the neutral stability conditions in Eqs. (10a-b).

\subsection{Element stiffness matrices}

From Eq. (17), by substituting field displacements into Eqs. (2a-h) and then Eq. (1) and evoking the stationarity condition, one obtains the stationarity condition

$\left([K]-\lambda\left[K_{G}\right]\right)\left\{\delta u_{n}\right\}=\{0\}$

element elastic stiffness matrix $[K]$ and geometric matrix $\left[K_{G}\right]$ are defined as

$$
\begin{aligned}
& {[K]=[K]_{f}+[K]_{s v}+[K]_{s}} \\
& {\left[K_{G}\right]=\left[K_{G}\right]_{N}+\left[K_{G}\right]_{M}+\left[K_{G}\right]_{V}+\left[K_{G}\right]_{q y}+\left[K_{G}\right]_{q z}}
\end{aligned}
$$

in which, the elastic stiffness matrices contributions $[K]_{f},[K]_{s v},[K]_{s}$ are due to flexural stresses, the Saint-Venant shear stresses and the remaining shear stresses, respectively and the 
geometric matrices contributions $\left[K_{G}\right]_{N},\left[K_{G}\right]_{M},\left[K_{G}\right]_{V},\left[K_{G}\right]_{q y},\left[K_{G}\right]_{q z}$ are due to normal forces, bending moments, shear forces, transverse load position effect and distributed axial load, respectively. These stiffness matrices are obtained from

$\left\{[K]_{f},[K]_{s v},[K]_{s},\left[K_{G}\right]_{N},\left[K_{G}\right]_{M},\left[K_{G}\right]_{V},\left[K_{G}\right]_{q y},\left[K_{G}\right]_{q z}\right\}=$
$\left[H^{-1}\right]_{8 \times 8}^{T}\left\{\left[M_{1}\right],\left[M_{2}\right],\left[M_{3}\right],\left[M_{4}\right],\left[M_{5}\right],\left[M_{6}\right],\left[M_{7}\right],\left[M_{8}\right]\right\}[H]_{8 \times 8}^{-1}$

in which closed-form expressions for matrices $\left[M_{1}\right]$ to $\left[M_{8}\right]$ have been provided in Appendix B.

\subsection{Stiffness matrix for the structure}

The element elastic and geometric matrices $[K]$ and $\left[K_{G}\right]$ are assembled to form the structure elastic and geometric matrices $[S]$ and $\left[S_{G}\right]$, respectively and the boundary conditions are enforced. In order to incorporate kinematic constraints into the formulation (such as the presence of eccentric supports, etc.), the structure is assumed to be subjected to a set of $m$ multiple point constraints of the form of

$[P]_{m \times n}\left\{u_{s}\right\}_{n \times 1}=\{0\}_{m \times 1}$

where, $[P]_{m \times n}$ is a matrix of user-input coefficients which linearly relate any set of nodal displacements, $\left\{u_{s}\right\}_{n \times 1}$ is the vector of nodal displacements for the structure and $n$ is the number of degrees of freedom. It is then required to extremize the second variation of the total potential energy $\pi$ of the system subject to the set of constraints in Eq. (21). This is formally achieved by introducing an auxiliary functional $\pi^{*}$ through augmenting the total potential energy $\pi$ by an additional term resulting from pre-multiplying the constraints in Eq. (21) by a vector of Lagrange multipliers $\langle F\rangle_{1 \times m}^{T}$ such that 


$$
\begin{aligned}
\delta\left(\frac{1}{2} \delta^{2} \pi^{*}\right) & =\delta\left(\frac{1}{2} \delta^{2} \pi+\langle F\rangle_{1 \times m}^{T}[P]_{m \times n}\left\{u_{s}\right\}_{n \times 1}\right) \\
= & \delta\left(\frac{1}{2}\left\langle u_{s}\right\rangle_{1 \times n}^{T}\left([S]_{n \times n}-\lambda\left[S_{G}\right]_{n \times n}\right)\left\{u_{s}\right\}_{n \times 1}+\langle F\rangle_{1 \times m}^{T}[P]_{m \times n}\left\{u_{s}\right\}_{n \times 1}\right)=0
\end{aligned}
$$

By evoking the stationarity conditions of the second variation of the functional $\pi^{*}, \partial\left((1 / 2) \delta^{2} \pi^{*}\right) / \partial\left\{u_{s}\right\}_{n \times 1}=\{0\}_{n \times 1}, \partial\left((1 / 2) \delta^{2} \pi^{*}\right) / \partial\{F\}_{m \times 1}=\{0\}_{m \times 1}$, one obtains

$$
\left\{\left[\begin{array}{ll}
{[S]_{n \times n}} & {[P]_{n \times m}^{T}} \\
{[P]_{m \times n}} & {[0]_{m \times m}}
\end{array}\right]-\lambda\left[\begin{array}{cc}
{\left[S_{G}\right]_{n \times n}} & {[0]_{n \times m}} \\
{[0]_{m \times n}} & {[0]_{m \times m}}
\end{array}\right]\right\}\left\{\begin{array}{l}
\left\{u_{s}\right\}_{n \times 1} \\
\{F\}_{m \times 1}
\end{array}\right\}=\left\{\begin{array}{l}
\{0\}_{n \times 1} \\
\{0\}_{m \times 1}
\end{array}\right\}
$$

The modified eigenvalue problem defined by Eq. (23) is then solved for the load multiplier $\lambda$, the associated eigen displacement vector $\left\{u_{s}\right\}_{n \times 1}$ and Lagrange multipliers $\{F\}_{m \times 1}$.

\section{Examples}

This section provides various buckling examples and evaluates the convergence characteristics of the proposed elements against other numerical solutions. Comparisons are also provided to the other solutions. The number of degrees of freedom needed to achieve convergence is established and the convergence behavior for the three element is discussed. The effects of lateral and torsional restraints and their location of lateral restraints relative to the shear center on lateraltorsional capacity are also assessed for simply supported and continuous multiple-span beams. In all examples, steel material is considered with $E=200,000 \mathrm{MPa}$ and $G=77,000 \mathrm{MPa}$. The W250x45 section is adopted (Fig. 3). Cross-sectional properties are $I_{x x}=71.887 \times 10^{6} \mathrm{~mm}^{4}, I_{y y}=7.033 \times 10^{6} \mathrm{~mm}^{4}, \quad A=5770.8 \mathrm{~mm}^{2}, \quad I_{\omega \omega}=1.124 \times 10^{11} \mathrm{~mm}^{6}$, $J=2.538 \times 10^{5} \mathrm{~mm}^{4}, D_{x x}=3848 \mathrm{~mm}^{2}$ and $D_{h h}=61.577 \times 10^{6} \mathrm{~mm}^{4}$. 


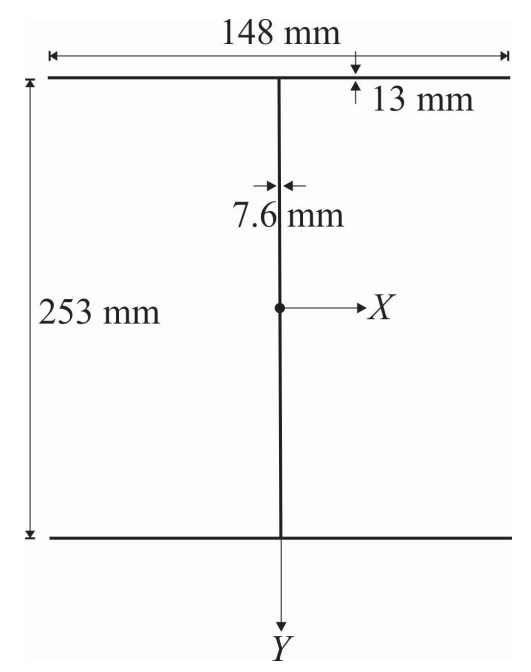

Fig. 3. Dimensions of the $W 250 \times 45$ cross-section

\subsection{Example 1: Mesh density analysis and comparison with other solutions for cantilevers}

A comparison is provided with solution developed by Wu and Mohareb [2] (WM element), the SM-M, SM-N and SM-X elements developed in the present study and the classical Barsoum and Gallagher element (referred to as BG element). The example was solved in [2] and is revisited here for comparison. It consists of a cantilever beam under a vertical concentrated load applied at the tip and acting at the shear center. Two spans are investigated; $5 \mathrm{~m}$ and $1 \mathrm{~m}$. A mesh study analysis is provided in Figs. 4 and 5. In Fig. 4, the horizontal line provides the converged value for the buckling load when a large number of elements are taken, i.e., $8 \mathrm{BG}$ elements (non-shear deformable) and $320 \mathrm{WM}$ elements (shear deformable). Both elements predicted the same critical load 41.3 KN within three significant digits since shear deformation effects are negligible for long spans. For the elements developed in the present study, 120 SM-X elements yielded a critical load prediction of $40.9 \mathrm{KN}$ while $120 \mathrm{SM}-\mathrm{N}$ elements yielded a value of $41.5 \mathrm{KN}$. The best and fastest prediction among the shear deformable elements was obtained using the SM-M element where 8 elements were enough to reach a critical load of $41.1 \mathrm{KN}$. As seen, results based on all four shear deformable elements (WM, SM-M, SM-N and SM-X) and the classical nonshear deformable BG element are in a close agreement for the beam with larger span. The SM-M element involves the smallest number of degrees of freedom (i.e., 8 elements) and unlike other displacement-based elements such as WM and BG elements, it is observed to converge from 
below in the present problem. On the other hand, the SM-N element converges from above at a relatively slow rate compared to SM-M, but still at a faster rate than the WM element. It is also observed that the resulting SM-X element converges from below again, albeit the convergence rate is in line with that of the SM-N element, but still faster than the WM element for the present example. For the $1 \mathrm{~m}$ span cantilever, Figure 5 shows that all four shear deformable elements (SM-N, SM-M, SM-X and WM) converge to the same critical load, while the classical shear non-deformable BG element is observed to slightly overestimate the buckling load, which is more pronounced in short span beams.

For comparison, a solution based on the ABAQUS S4R shell element as reported in [2] was also provided. The ABAQUS S4R solution yielded buckling load estimates of $39.98 \mathrm{kN}$ and $2396 \mathrm{kN}$ for the long and short span members, respectively. Both predictions are slightly lower than those based on the shear deformable elements. The difference is attributed to the fact that the shell element captures both the distortional and shear deformation effects and thus provides the most flexible representation of all solutions. The largest buckling load observed is that based on the BG element. This is expected since the BG element neglects distortional and shear deformation effects and thus provides the stiffest representation of the member.

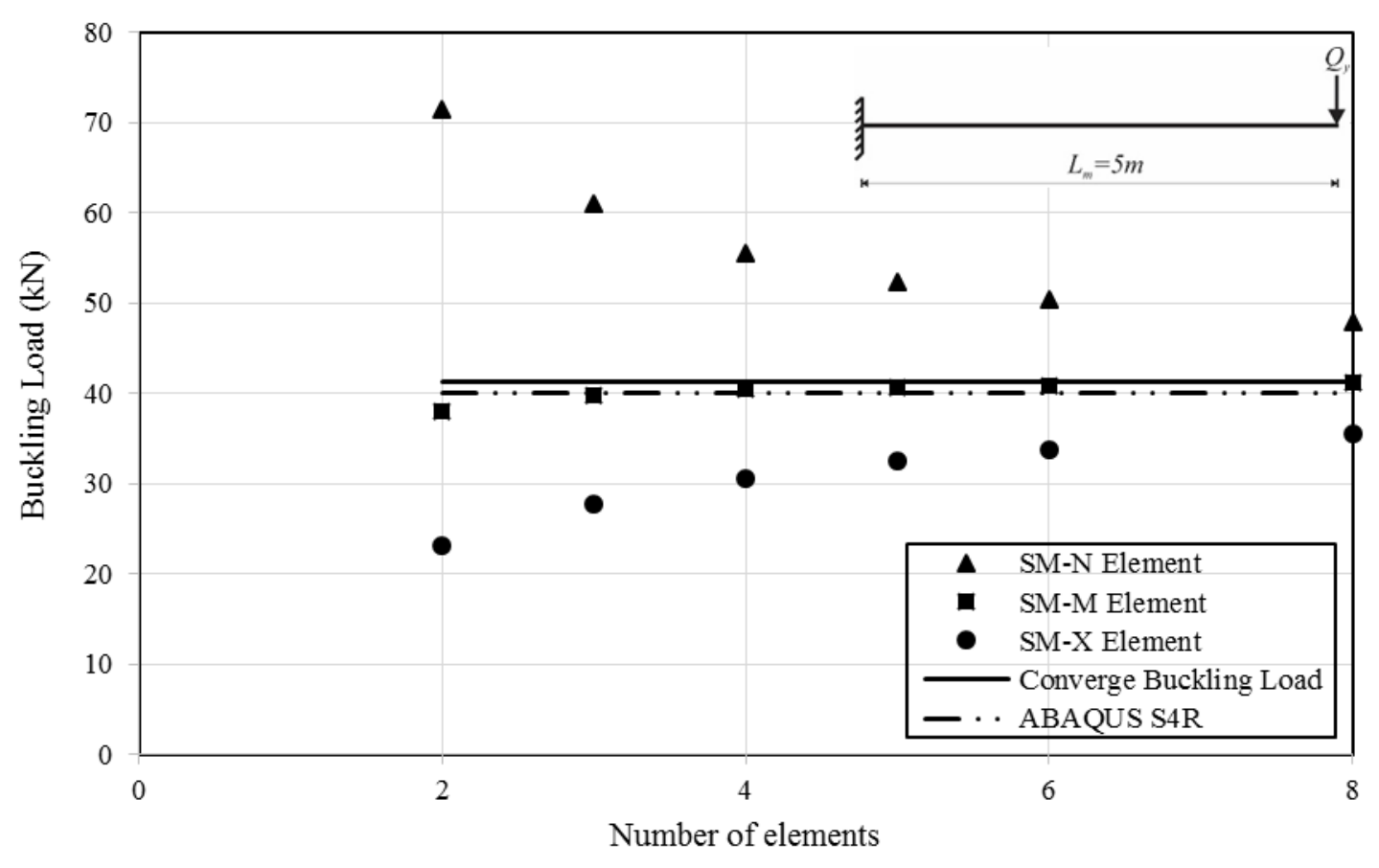


Fig. 4. Mesh study analysis for the cantilever beam with larger span

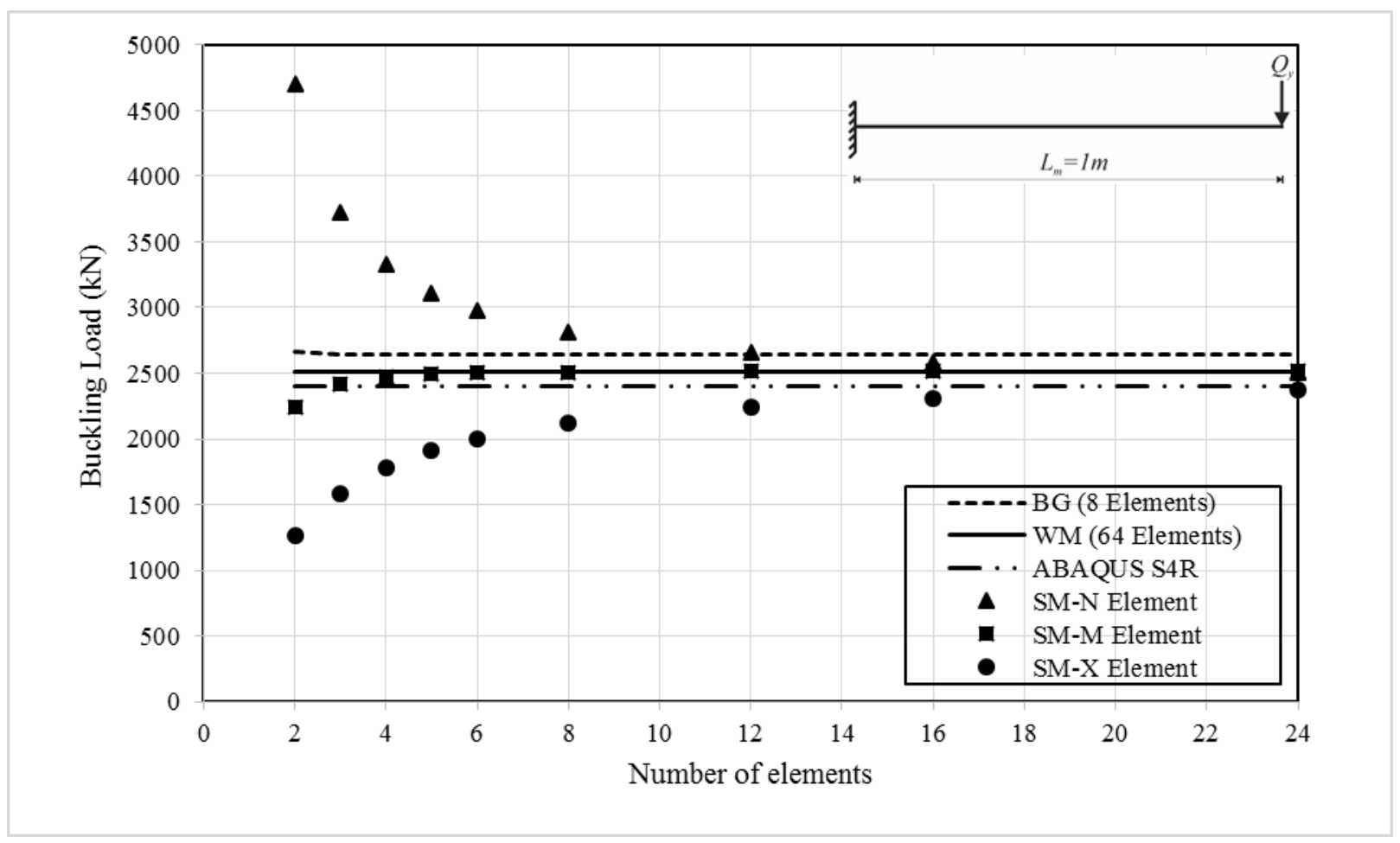

Fig. 5. Mesh study analysis for the cantilever beam with shorter span

\subsection{Example 2: Convergence characteristics for other loading conditions}

While Example 1 has shown the convergence characteristics of the elements SM-N, SM-M and SM-X for the particular case of a cantilever under tip load, the present example is aimed to investigate the convergence characteristics for other types of loading. Four additional loading cases are considered for a $5 \mathrm{~m}$ span simply supported beam under the following loads.
a) Mid-span point load,
b) Uniformly distributed load,
c) Uniform bending moments, and
d) Linear reverse moments.

A mesh density study was performed for each of the five cases using each of the three elements. The results are summarized in Table 1 for SM-M element, Table 2 for SM-N element and Table 3 for SM-X elements. In all five cases, the SM-M is observed to be fast converging compared to 
the other two elements. Mesh refinements from 8 to 120 elements were associated with a difference in the predicted buckling moments of less the $2 \%$ in all five cases. The solution is observed to converge from below for the cases of reverse moments and cantilever and from above in other cases, i.e., no specific convergence trend can be guaranteed under the SM-M element.

For element SM-N, Table 2 indicates that convergence is from above in all cases. The number of elements needed for convergence is higher than the SM-M, where 48 elements were associated with a $3 \%$ difference compared to solutions based on 120 elements.

For element SM-X, Table 3 indicates that convergence is from below in all cases, i.e., discertization errors consistently result in an underestimation of the buckling moments. This feature is desirable from a design viewpoint, since it consistently errs on the conservative side. Solutions based on 48 elements are observed to agree within 3\% of those based on 120 elements. The present study suggests that both elements SM-N and SM-X are comparatively more computationally efficient than the WM element [2], where 320 elements have been reported to be needed for convergence. While elements SM-N and SM-X appear to consistently provide upper and lower bounds of the buckling moments, element SM-M is most efficient in terms of computational effort required for convergence. A comparison for the results of SM-X, SM-M and SM-N elements are provided in Table 4. When the same number of elements are taken (120 elements in the present study), the buckling moments predicted by SM-M element are observed to always lie between those based on SM-X and SM-N elements. Results based on SM-X thus appear to provide lower bound predictions while those based on SM-N provide upper bound predictions of the buckling moments.

Table 1 - Buckling moments (KNm) and convergence characteristics predicted by SM-M element

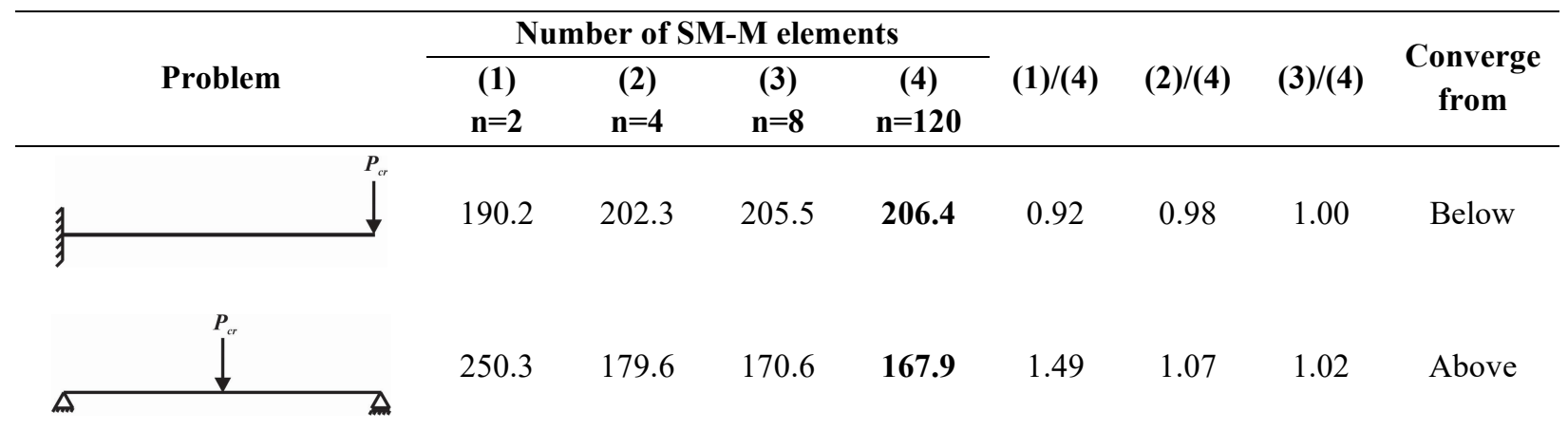




\begin{tabular}{rrrrrrrrrr}
\hline \\
\hline$M_{c r}$
\end{tabular}

Table 2 - Buckling moments (KNm) and convergence characteristics predicted by SM-N element

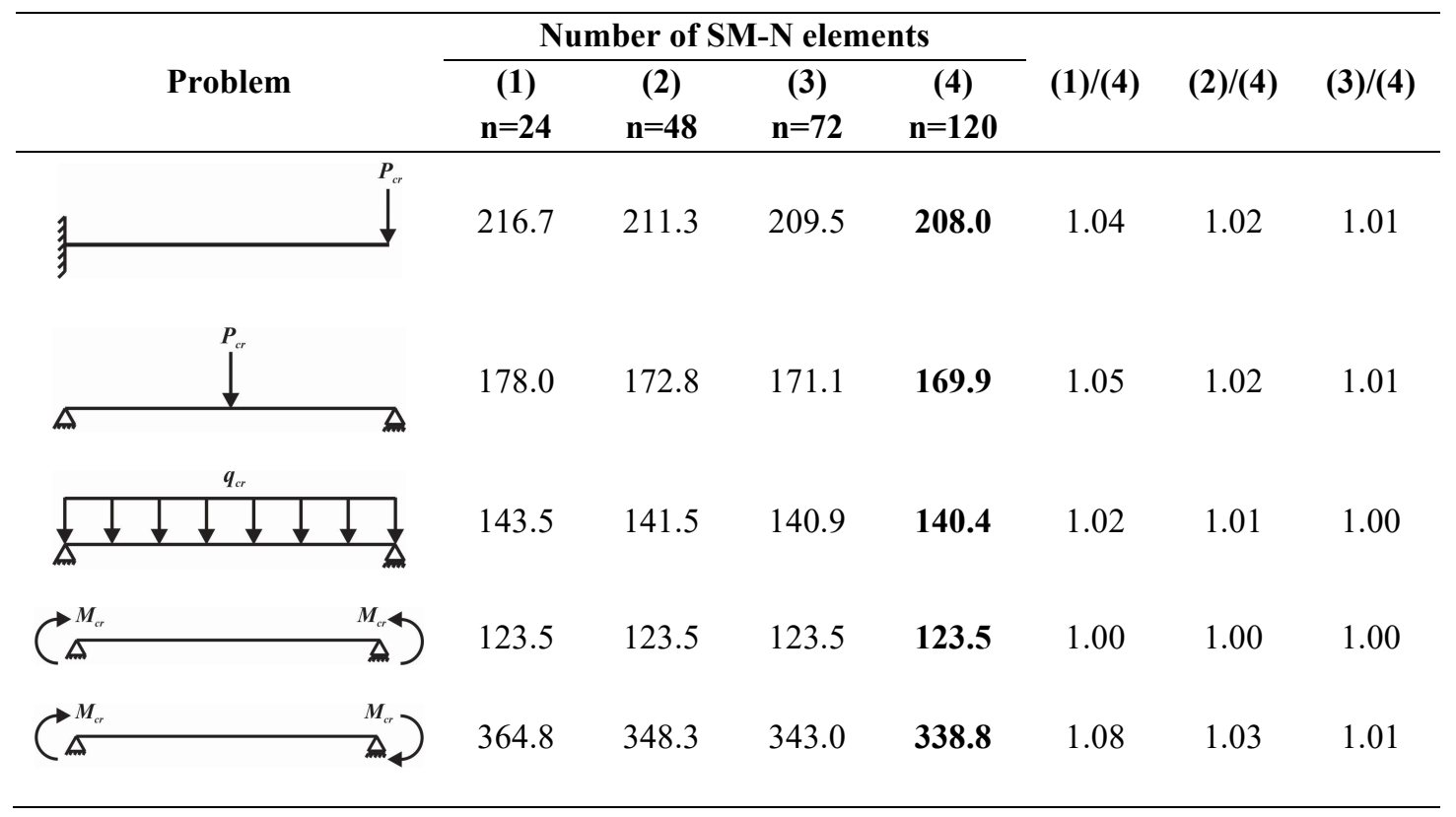

Table 3 - Buckling moments (KNm) and convergence characteristics predicted by SM-X element

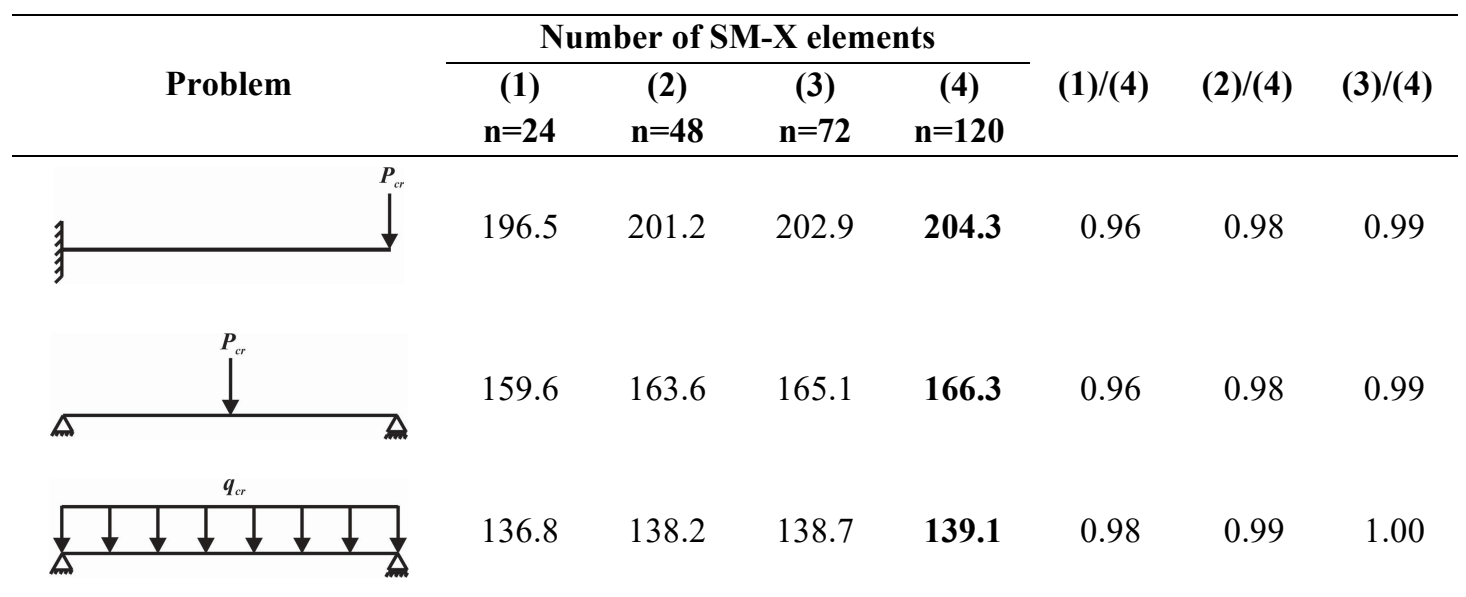




\begin{tabular}{|c|c|c|c|c|c|c|c|c|}
\hline$C_{\Delta}^{M_{c r}}$ & $\begin{array}{c}M_{c t} \rightarrow \\
\Delta\end{array}$ & 123.5 & 123.5 & 123.5 & 123.5 & 1.00 & 1.00 & 1.00 \\
\hline$C_{\Delta}^{M_{c r}}$ & 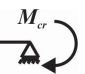 & 304.2 & 318.1 & 322.9 & 326.8 & 0.93 & 0.97 & 0.99 \\
\hline
\end{tabular}

Table 4 - Comparison between buckling moments $(\mathrm{KNm})$ predicted by SM-X, SM-M and SM-N elements (120 elements were taken in all cases)

\begin{tabular}{|c|c|c|c|}
\hline Problem & $\begin{array}{c}\text { SM-X } \\
\text { Lower Bound }\end{array}$ & SM-M & $\begin{array}{c}\text { SM-N } \\
\text { Upper Bound }\end{array}$ \\
\hline & 204.3 & 206.4 & 208.0 \\
\hline ․ & 166.3 & 167.9 & 169.9 \\
\hline 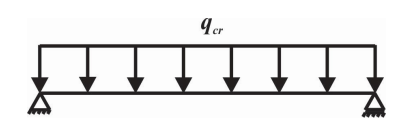 & 139.1 & 139.6 & 140.4 \\
\hline $\begin{array}{c}M_{c c} \\
\Delta\end{array}$ & 123.5 & 123.5 & 123.5 \\
\hline$C_{\Delta}^{M_{\sigma r}}$ & 326.8 & 332.7 & 338.8 \\
\hline
\end{tabular}

\subsection{Example 3: Effect of lateral and torsional restraints on buckling capacity of beams}

A $4 \mathrm{~m}$ span simply supported beam with a W250x45 cross-section is subject to reverse end moments as shown in Fig. 6. Five bracing scenarios were considered: Case 1 involved a lateral restraints at shear center $\left(u_{b}=0\right)$, Case 2 involved a lateral restraint at the bottom flange i.e., $\left[\theta_{z b}(d / 2)-u_{b}=0\right]$, Case 3 involved a lateral restraint at the top flange, i.e., $\left[\theta_{z b}(d / 2)+u_{b}=0\right]$, Case 4 involved a torsional restraint, i.e., $\left(\theta_{z b}=0\right)$ and Case 5 considered torsional and lateral restraints at shear center, i.e., $\left(u_{b}=0, \theta_{z b}=0\right)$. All constraints were used to form the matrix of coefficients $[P]$ introduce in Eqs. (21)-(23) and the resulting constrained eigenvalue problem was solved to yield the critical load combinations. Results are illustrated in 
Table 5. For each scenario, five solutions based on SM-X, SM-M, SM-N, BG elements and ABAQUS B31OS element are provided.

According to Abaqus documentation ${ }^{\dagger}, \mathrm{B} 31 \mathrm{OS}$ is listed among Timoshenko-type beam elements, in which the flexural shear stiffness values are internally computed from user-input section geometries. It is the authors' experience (e.g., [49]) that shear deformation effects within the element are limited to flexural shear and the element omits shear deformation effects due to nonuniform warping.

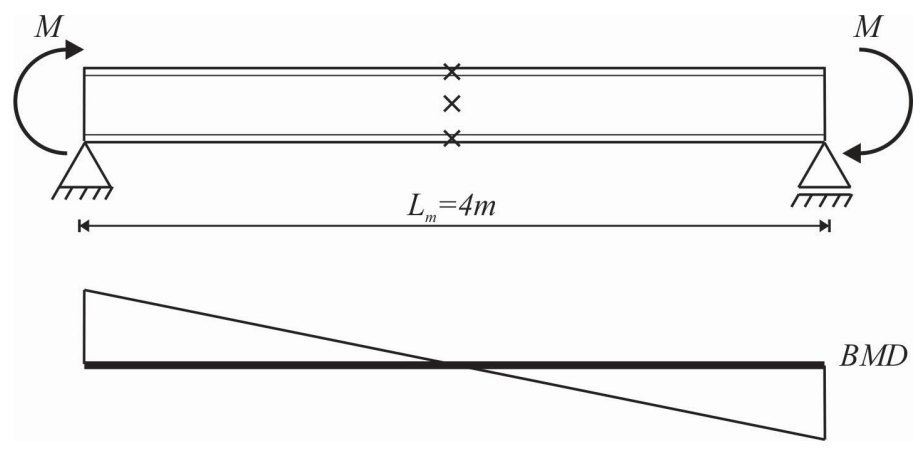

Fig. 6. Simply supported beam restrained at mid-span subject to reverse end moments

Table 5 - Critical moments (KNm) for various mid-span constraints

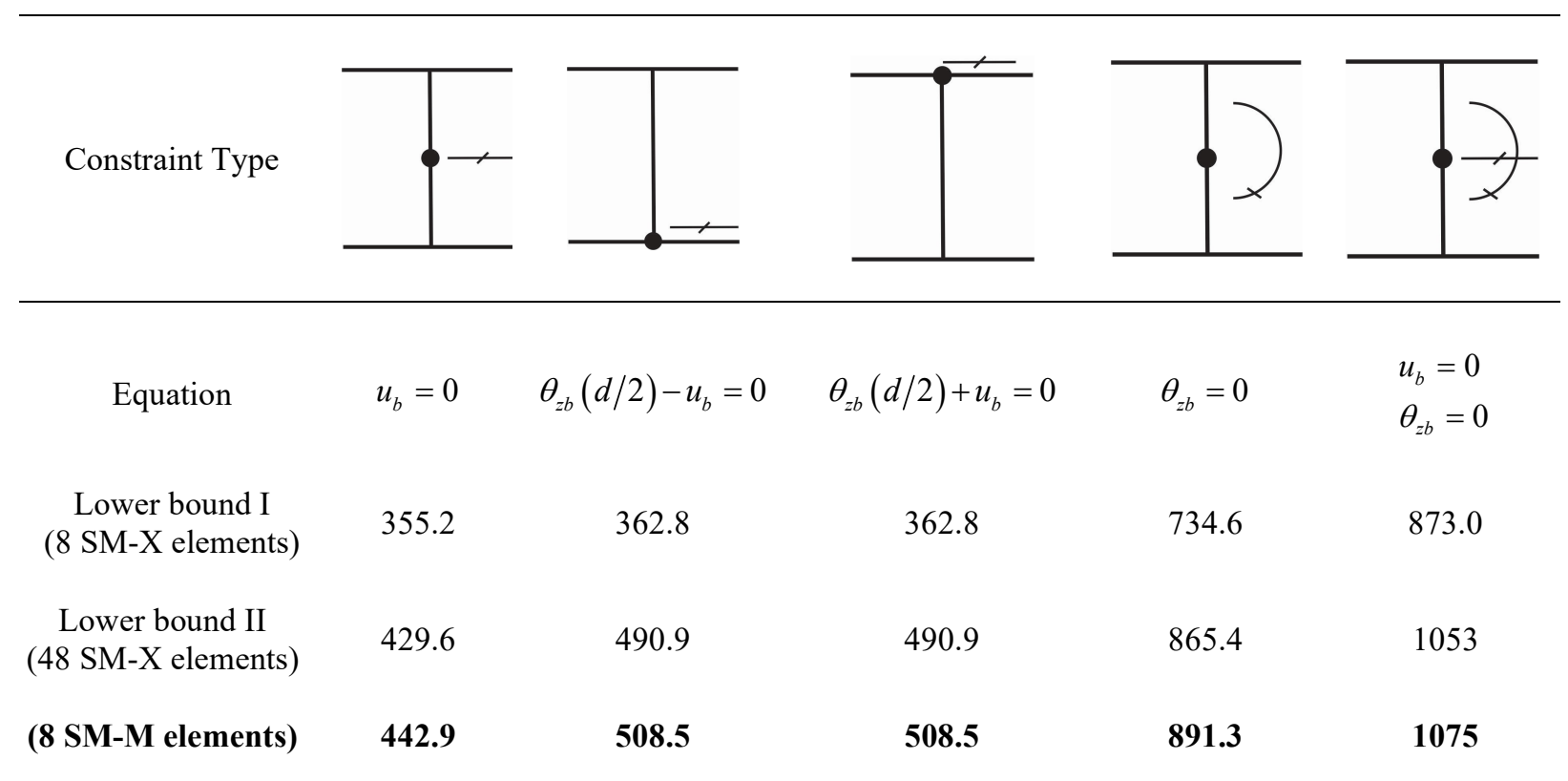

${ }^{\dagger}$ Abaqus 6.12, Analysis User’s Manual, Volume VI: Elements, Section 29.3.3-3 


\begin{tabular}{cccccc}
$\begin{array}{c}\text { Upper bound I } \\
\text { (48 SM-N elements) }\end{array}$ & 470.6 & 539.1 & 539.1 & 929.3 & 1115 \\
$\begin{array}{c}\text { Upper bound II } \\
\text { (8 SM-N elements) }\end{array}$ & 591.8 & 676.8 & 676.8 & 1123 & 1290 \\
$\begin{array}{c}\text { ABAQUS B31OS } \\
\text { (40 elements) }\end{array}$ & 451.7 & 518.2 & 518.2 & 906.6 & 1099 \\
BG (8 elements) & 452.7 & 519.4 & 519.4 & 908.8 & 1106 \\
SM-M/B31OS & $\mathbf{9 8 . 0 \%}$ & $\mathbf{9 8 . 1 \%}$ & $\mathbf{9 8 . 1 \%}$ & $\mathbf{9 8 . 3 \%}$ & $\mathbf{9 8 . 0 \%}$ \\
\hline
\end{tabular}

Results based on the SM-X, SM-M and SM-N elements (which capture shear deformation due to bending and warping) are compared with those predicted by the ABAQUS B31OS element (which captures shear deformation effects due to bending, but neglects shear deformation effects due to warping) and those based on the BG element (neglecting both shear deformation effects due to bending and warping).

Eight elements were needed for convergence under the SM-M and BG solutions while 40 B31OS elements were needed to attain convergence. Results based on the SM-M are shown to agree well with those based on the B31OS solution.

On average, the SM-M solution is observed be about $2.0 \%$ less than the B31OS solution and $2.2 \%$ less than the BG solution. The difference is attributed to shear deformation which is entirely captured in the SM-M element, only in part in the B31OS, and neglected in the BG element. In all cases, taking $8 \mathrm{SM}-\mathrm{N}$ and $8 \mathrm{SM}-\mathrm{X}$ elements is observed to respectively provide upper and lower bounds for the 8 SM-M solutions. Also, improved upper and lower bounds were observed by taking 48 SM-N and SM-X elements, thus narrowing the band between both bounds.

Restraining the bottom or top flanges (Cases 2 and 3) laterally is observed to increase the buckling capacity of the beam more than the case where the shear center is laterally restrained (Case 1). As expected, restraining the shear center both laterally and torsionally (Case 5) is found to significantly increase the buckling load by more than twice the capacity compared to the case where no restraints are provided. Restraining the section torsionally (Case 4) is observed to be more effective in increasing the buckling capacity compared to cases where either the shear center or one of the flanges is laterally restrained (Cases 1-3). Figure 7 depicts the lateral 
buckling displacements for the top and bottom flanges for all five cases as calculated by the expressions $u_{b} \pm \theta_{b}(h / 2)$ versus the longitudinal coordinate $z$. In all cases, the buckling mode shapes were normalized with respect to the peak flange displacement. In Cases 1 and 5 , the average top and bottom displacements (or mid-height) at mid-span, vanishes given the presence of a lateral restraint at mid-height. Also, for Cases 4 and 5, the top and bottom flange displacements are equal since twist was restrained in both cases. For Case 2, it is clear that the bottom flange is successfully restrained at mid-span. Also, in Case 3, the mid-span top flange was restrained. In Case 1, the peak flange displacements take place near $z=1.5 \mathrm{~m}$ and $2.5 \mathrm{~m}$. It is also shown that, when one of the flanges is laterally restrained (Cases 2 and 3), the peak lateral displacement occurs at about $z=2.5 \mathrm{~m}$. The maximum lateral displacements for Cases 4 and 5 are observed at nearby $z=1 \mathrm{~m}$ and $z=3 \mathrm{~m}$.

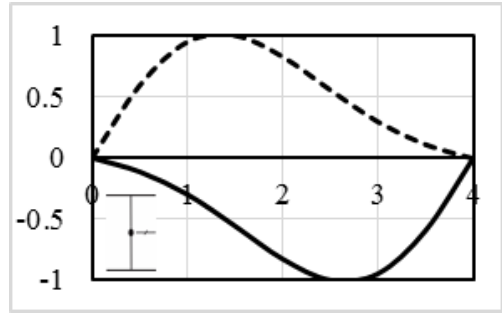

(a)

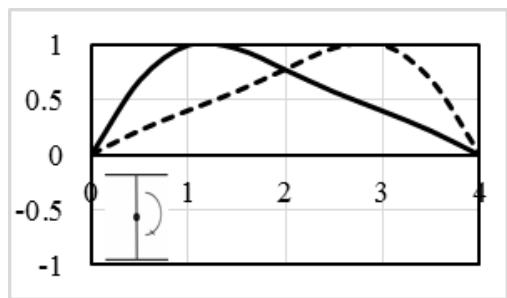

(d)

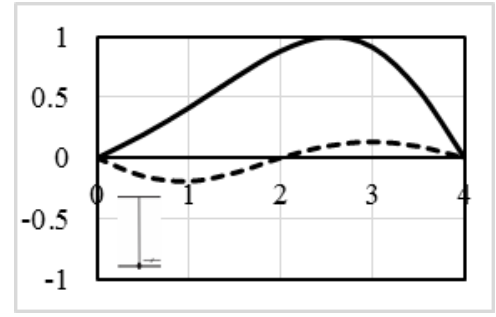

(b)

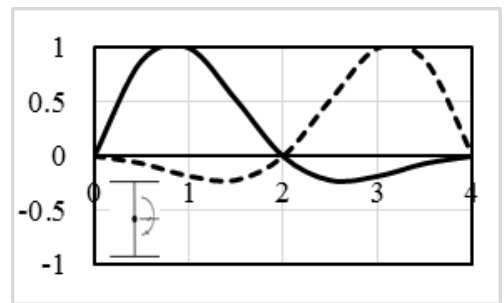

(e)

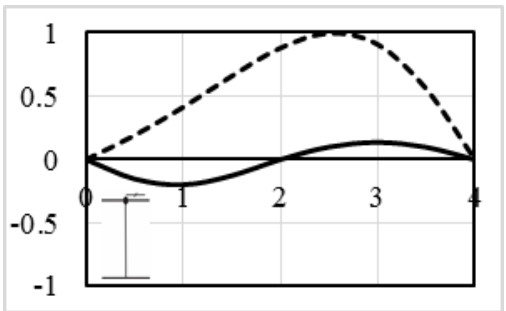

(c)

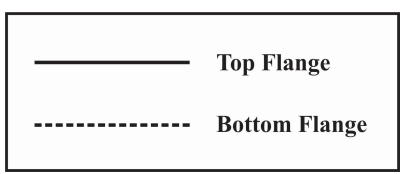

Fig. 7. Normalized lateral displacement of the top flange and the bottom flange along the span (m) for various mid-span constraints: (a) Case 1, (b) Case 2, (c) Case 3, (d) Case 4 and (e) Case 5 


\subsection{Example 4: Effect of the lateral brace height on the critical moment}

Among studies present in the literature review, the work of [9, 14-16, 23] investigated the effect of rigid lateral or torsional discrete intermediate restraints on LTB capacity of beams. Lateral restraints in these studies were assumed to act at the shear center. In practice, I-girders can be laterally restrained at various locations along the height. Thus, the present example aims at quantifying the effect of bracing height on the LTB resistance of beams. The simply supported beam investigated in the previous example is revisited here while considering mid-span lateral restraints located at various heights $h$ relative to the shear center within the range $-d / 2 \leq h \leq d / 2$, in which $d$ is the depth of cross-section (Fig. 8). The resulting critical moments are normalized with respect to the solution based on $h=0$ (i.e., the case of shear center lateral restraint). For the case of the reverse moments, the most effective bracing location is found to be at either flange while mid-height bracing is observed to be the least effective. The case of flange bracing corresponds to a $15 \%$ increase in the critical moments when compared to that of midheight bracing.

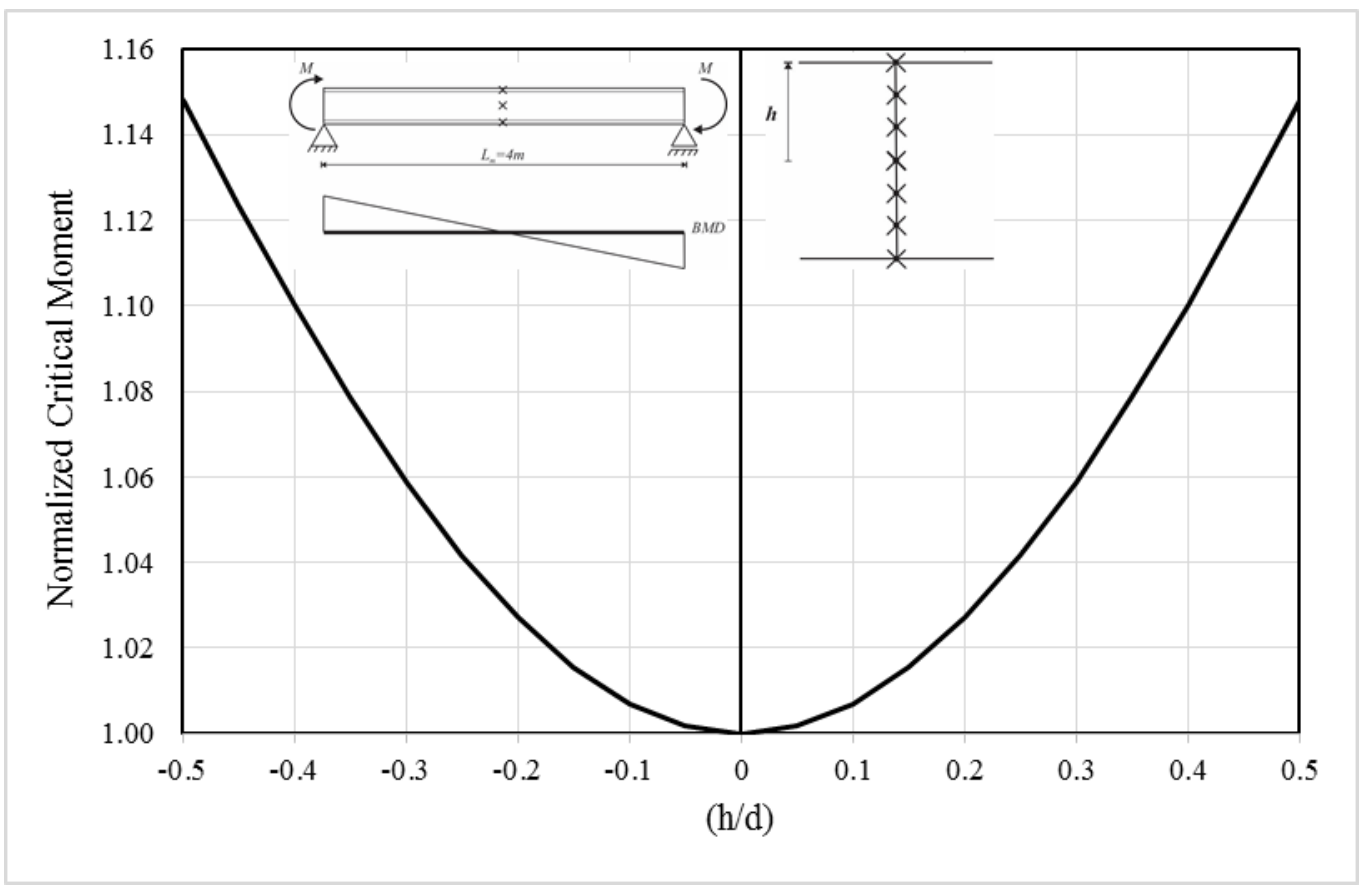

Fig. 8. Effect of constraint's location on critical moment for a simply supported beam of $4 \mathrm{~m}$ span 


\subsection{Example 5: Effect of mid-span restraints on buckling capacity of continuous beams}

An evaluation of the effect of lateral and torsional restraints at the interior supports on the buckling capacity of a continuous beam is practical importance. A two-span continuous beam with $8 \mathrm{~m}$ spans [Fig. 9(b)] is investigated in this example. As a reference case for comparison, another $8 \mathrm{~m}$ span simply supported beam is also considered [Fig. 9(a)]. Both beams are subjected to uniformly distributed loads. Four cases of lateral and/or torsional restraints are considered for the interior support [(Fig. 9(c)]: (1) No lateral nor torsional restraints, (2) only a lateral restraint at the shear center, (3) only a torsional restraint, and (4) both lateral and torsional restraints.

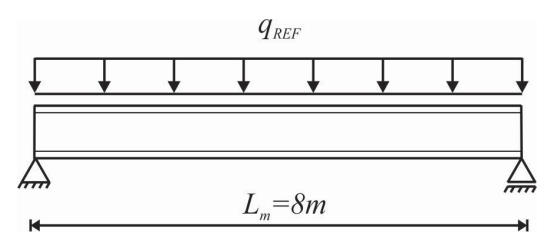

(a)

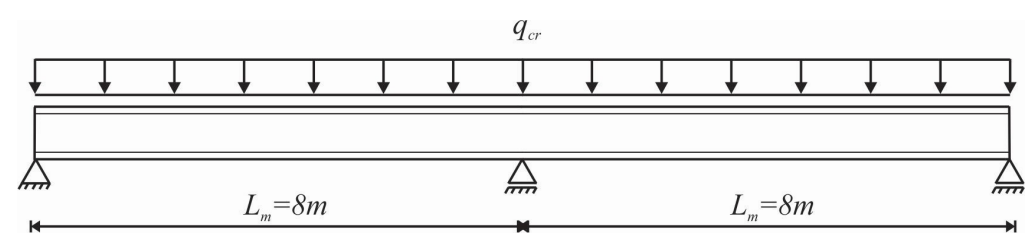

(b)

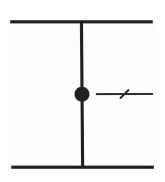

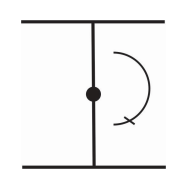

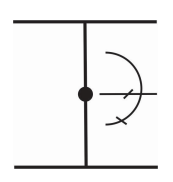

(c)

Fig. 9. (a) Reference case, (b) Multi-span beam used for cases (1) to (4) and (c) Cross-sections at middle support for various cases (1) to (4)

Table 6 provides the critical loads based 8 SM-M elements for each span. The critical load of the continuous two-span beam with no lateral nor twist restraint at the intermediate support (Case 1) is $8.9 \mathrm{KN}$ which is $12 \%$ less than that of the simply supported beam. The presence of a lateral support at the middle support (Case 2) is observed to marginally increase the buckling capacity (i.e., by $2 \%$ ) of the continuous beam compared to the unrestrained case (Case 1 ). In contrast, the presence of a torsional brace (Case 3 ) is observed to significantly increase the lateral-torsional 
capacity compared to Case 1. The presence of both restraints (lateral and torsional) in Case 4 yields the same critical moment as that in Case 3. The comparison suggests that the presence of a torsional restraint at the intermediate support is most effective in increasing the LTB resistance of the continuous beam considered.

For the simply supported beam (i.e., the reference case), the critical load as predicted by $8 \mathrm{SM}-\mathrm{M}$ elements is $10.1 \mathrm{KN} / \mathrm{m}$. Taking $8 \mathrm{SM}-\mathrm{X}$ elements provides a lower bound prediction of 9.39 $\mathrm{KN} / \mathrm{m}$ for the critical load, while $8 \mathrm{SM}-\mathrm{N}$ elements provides an upper bound prediction of 10.9 $\mathrm{KN} / \mathrm{m}$. As observed in Example 3, increasing the number of elements leads to improved lower and upper predictions. For instance, by taking 24 elements, the critical load predictions is bounded between $9.69 \mathrm{KN} / \mathrm{m}$ and $10.2 \mathrm{KN} / \mathrm{m}$.

Table 6 - Summary of buckling loads for the reference case and cases (1) to (4) - Based on SM-M element

\begin{tabular}{ccccccc}
\hline & \# of Span(s) & $\begin{array}{c}\text { Lateral } \\
\text { restraint }\end{array}$ & $\begin{array}{c}\text { Torsional } \\
\text { restraint }\end{array}$ & $q_{c r}(K N / m)$ & $q_{R E F}(K N / m)$ & $q_{c r} / q_{R E F}$ \\
\hline Reference Case & 1 & N/A & N/A & - & 10.1 & 1.0 \\
Case (1) & 2 & $\mathbf{x}$ & $\mathbf{x}$ & 8.90 & - & 0.88 \\
Case (2) & 2 & $\checkmark$ & $\mathbf{x}$ & 9.10 & - & 0.90 \\
Case (3) & 2 & $\mathbf{x}$ & $\checkmark$ & 20.2 & - & 2.0 \\
Case (4) & 2 & $\checkmark$ & $\checkmark$ & 20.2 & - & 2.0 \\
\hline
\end{tabular}

\subsection{Example 6: Effect of shear deformation}

The cantilever beam problem in Example 1 is re-considered. The span is varied from $0.2 \mathrm{~m}$ to 3.0m. The critical loads are obtained based on the SM-M, B31OS and BG elements. The resulst are illustrated in Table 7 and depicted in Fig. 10.

Table 7 - Critical Loads $\mathrm{f} \boldsymbol{P}_{c r}(\boldsymbol{k N})$ or various spans 


\begin{tabular}{|c|c|c|c|}
\hline $\mathbf{L}$ (m) & $\begin{array}{c}\text { SM-M } \\
\text { Element }\end{array}$ & $\begin{array}{c}\text { B31OS } \\
\text { Element }\end{array}$ & $\begin{array}{c}\text { BG } \\
\text { Element }\end{array}$ \\
\hline 0.2 & 135,400 & 150,000 & 292,800 \\
\hline 0.4 & 28,820 & 29,580 & 37,250 \\
\hline 0.6 & 10,010 & 10,140 & 11,340 \\
\hline 0.8 & 4,598 & 4,640 & 4,955 \\
\hline 1.0 & 2,510 & 2,528 & 2,640 \\
\hline 1.2 & 1,536 & 1,546 & 1,594 \\
\hline 1.4 & 1,019 & 1,025 & 1,049 \\
\hline 1.6 & 716.3 & 720.8 & 733.7 \\
\hline 1.8 & 526.8 & 530.11 & 537.7 \\
\hline 2.0 & 401.2 & 403.7 & 408.5 \\
\hline 2.2 & 314.2 & 316.2 & 319.3 \\
\hline 2.4 & 251.8 & 253.4 & 255.4 \\
\hline 2.6 & 205.6 & 206.9 & 208.3 \\
\hline 2.8 & 170.6 & 171.7 & 172.7 \\
\hline 3.0 & 143.5 & 144.4 & 145.2 \\
\hline
\end{tabular}

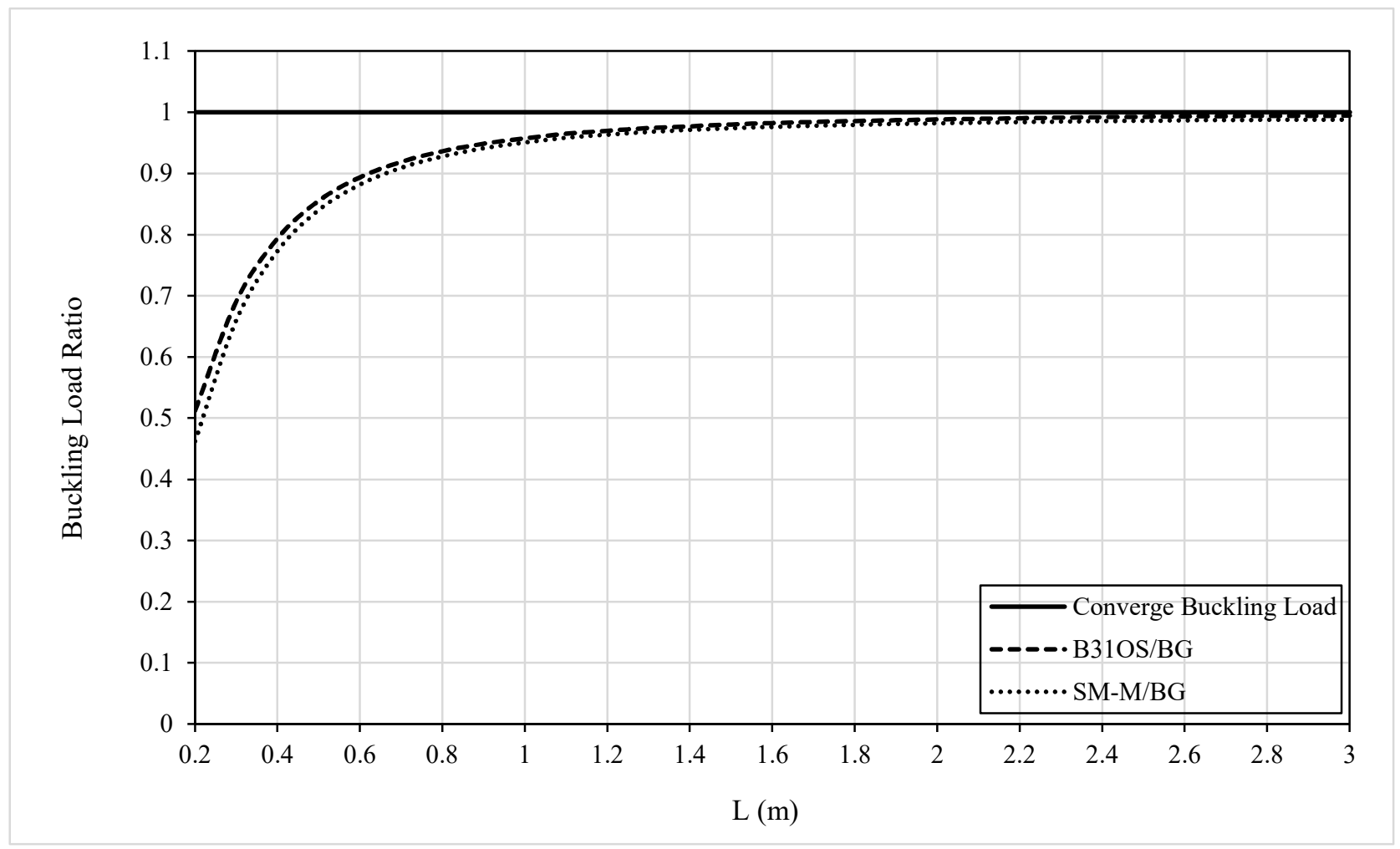

Fig. 10. Normalized LTB loads for the cantilever example 
As the span decreases, the difference between the non-shear-deformable BG element predictions and the SM-M predictions is observed to increase since shear deformation effects become pronounced for shorter spans. The SM-M predictions are observed to be slightly below the shear deformable critical load predictions of the B31OS element. As discussed in Example 3, this is attributed to the fact that the B31OS element captures the effect of shear deformation due to bending, but omits shear deformation effects due to warping. In contrast, both features are incorporated in the SM-M formulation. For spans shorter than $0.6 \mathrm{~m}$, the ratio of the critical loads based on the SM-M element to those based on the BG element is less than $90 \%$, highlighting the importance of shear deformation effects for short span members.

\section{Summary and conclusions}

1. A new family of three finite elements was developed for the lateral-torsional buckling analysis of beams with doubly-symmetric cross-sections. The elements capture warping torsion, shear deformation, and load position effects.

2. The results based on the three elements were in an excellent agreement with those based on shear deformable WM element [2] and successfully avoid shear locking phenomena. Very good agreement is also observed with the B31OS ABAQUS element and the nonshear deformable Barsoum and Gallagher [8] element for long span members.

3. A large number of numeric examples have shown that two of the elements are successful in bounding buckling load estimates. In all cases, mesh refinements are observed in narrowing the bounds for the predicted buckling load.

4. The SM-M element is observed to provide the fastest converging solution with a remarkably small number of degrees of freedom compared to the SM-N and SM-X and WM shear deformable element [2] and is thus recommended if the analyst is seeking computational efficiency.

5. Within the limitations of the formulation, discretization errors in the SM-X element consistently provide lower bound estimates for the buckling loads. Element SM-X is recommended for design situations where the designer would rather err on the conservative side. 
6. For the simply supported beam under full reverse moments considered, it was shown that torsional restraint at mid-span is most effective in increasing the LTB capacity compared to shear center or flange lateral restraints. Providing torsional and lateral restraints was observed to increase the buckling capacity by more than twofold compared to the case of no lateral and torsional restraints.

7. For the same problem, a brace at one of the flanges was shown to increase the LTB capacity of the beam by $15 \%$ compared to the case of web mid-height bracing.

8. At intermediate supports of two-span beams, the presence of torsional restraints was observed to significantly improve the lateral-torsional buckling capacity of continuous beams compared to lateral restraints and compared to the case of no lateral nor torsional restraints.

\section{Acknowledgements}

The authors gratefully acknowledge financial support from the Natural Sciences and Engineering Research Council (NSERC) of Canada. 


\section{Appendix A. Evaluating the simplifying assumption made to develop shape functions}

This section provides a numeric example assessing the assumption made in formulating shape functions regarding the neglect of pre-buckling internal forces. Assuming constant pre-buckling internal as shown in Fig. 2(b), Eq. (5) can be rewritten in a non-dimensional form as follows

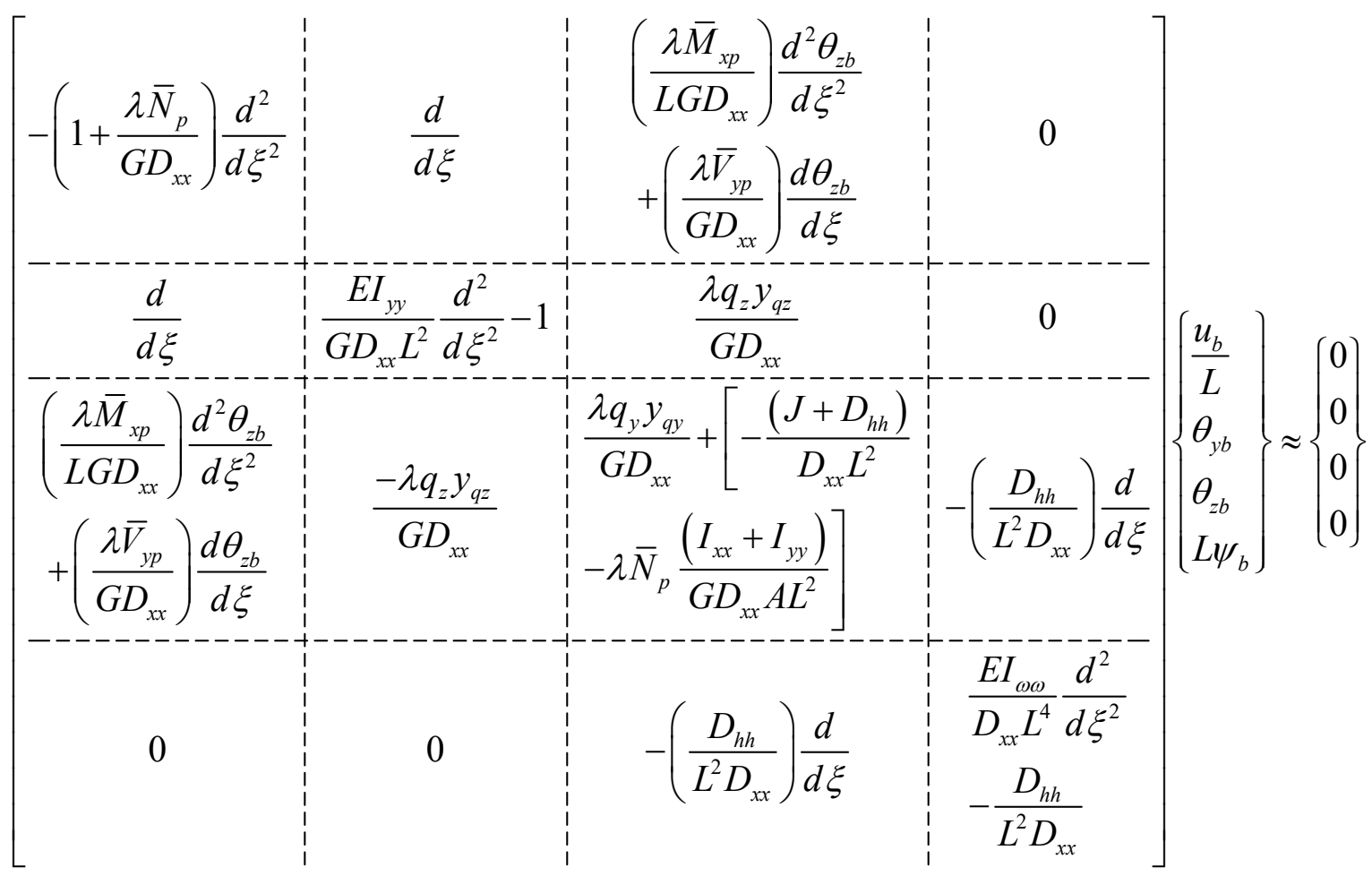

(A1)

in which, $D=d / d z=d / L d \xi$ is the first derivative of displacement fields with respect to the dimensionless coordinate $\xi=z / L$. It can be shown that for practical geometries, the term $\lambda \bar{N}_{p} / G D_{x x}$ in the first entry of the first equation in Eq. (A1), is negligible compared to unity. Also, the terms $\lambda \bar{M}_{x p} / L G D_{x x}$ and $\lambda \bar{V}_{y p} / G D_{x x}$ in the third entry of the first equation are also negligible compared to the zero value. In a similar manner, the term located in the third row and third column including $\lambda \bar{N}_{p}\left(I_{x x}+I_{y y}\right) / G D_{x x} A L^{2}$ is found insignificant compared to $\left(J+D_{h h}\right) / D_{x x} L^{2}$. To numerically illustrate the above statements for practical problems, consider a simply supported member (cross-section is W250x45 and member span is taken as $L_{m}=120 r_{y}=4189 \mathrm{~mm}$ ), subject to a compressive axial force [Case (a) in Fig. A.1] and uniform 
bending moments [Case (b) in Fig. A.1]. To get an indication of the magnitude of the shear force involved, we also consider another loading case [Case (c) in Fig. A.1] which involves no axial force but equal reverse moments (thus inducing a linear moment gradient, or constant shear). Since no transverse forces offset from the shear center and no axial forces offset from the section centroid are applied, the terms $\left(\lambda q_{y} y_{q y}\right)$ and $\left(\lambda q_{z} y_{q z}\right)$ are omitted.

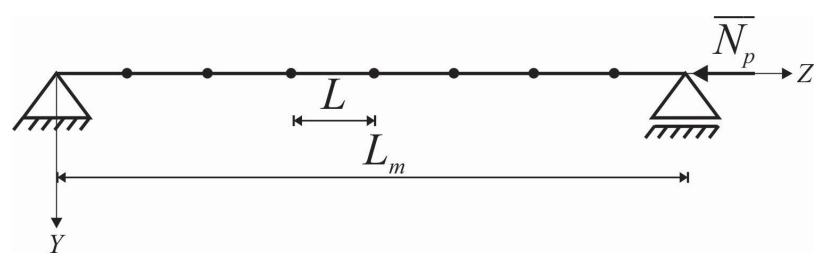

(a)

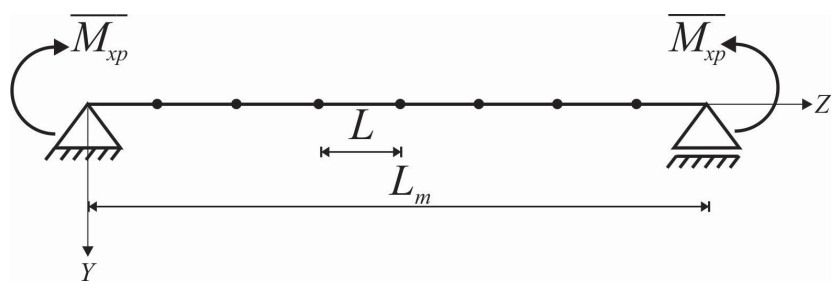

(b)

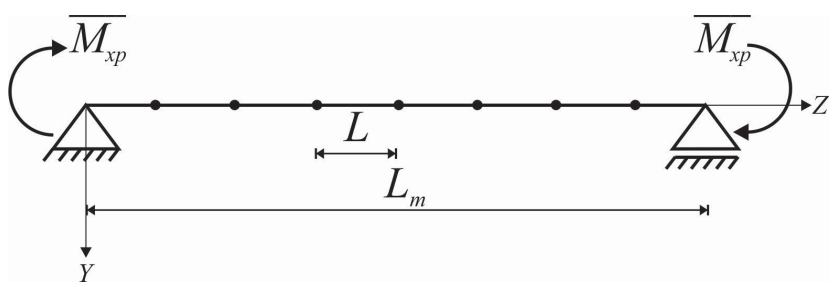

(c)

Fig. A.1. (a) Elevation of the beam under axial load, (b) Elevation of the beam under uniform bending moments, (c) Elevation of the beam under reverse bending moments

In Case (1), in the absence of bending moments, the buckling load predicted by the classical solution, would be $\bar{N}_{p}=\pi^{2} E I_{y y} / L_{m}^{2}=791 K N$. Also, in Case (2) in the absence of axial force and according to the classical solution, the critical buckling moment of the beam would be $\bar{M}_{x p}=\pi / L_{m} \sqrt{E I_{y y}\left(G J+E I_{\omega \omega} \pi^{2} / L_{m}^{2}\right)}=160 K N m$. For Case (3), the critical moment is given by $\lambda \bar{M}_{x p}=C_{b} \lambda \bar{M}_{x p}$, where $C_{b}$ is a moment gradient factor $\left(C_{b}=2.27\right.$ for this case) given by AISC 
[50], $C_{b}=\left(12.5 M_{\max }\right) /\left(2.5\left|M_{\max }\right|+3\left|M_{L / 4}\right|+4\left|M_{L / 2}\right|+3\left|M_{3 L / 4}\right|\right)$, in which $M_{\max }$ is the maximum bending moment along the beam, $M_{L / 4}, M_{L / 2}$ and $M_{3 L / 4}$ are bending moments at the quarter, mid-point and three-quarter points of the beam span, respectively. The corresponding shear is given by $\lambda \bar{V}_{y p}=2 \lambda \bar{M}_{x p} / L_{m}=2 C_{b} \lambda \bar{M}_{x p} / L_{m}=173 \mathrm{KN}$. Table A.1 summarizes the numeric values and corresponding approximations considered for the relevant matrix entries.

Table A.1 - Justification of assumption made to obtain shape functions

\begin{tabular}{|c|c|c|c|}
\hline Entry* & Expression & Values and Comments & Approximation \\
\hline 1,1 & $-\left(1+\frac{\lambda \bar{N}_{p}}{G D_{x x}}\right) \frac{d^{2}}{d \xi^{2}}\left(\frac{u_{b}}{L}\right)$ & $\begin{array}{c}\lambda \bar{N}_{p} / G D_{x x}=2.7 \times 10^{-3} \\
\text { (negligible compared to unity) }\end{array}$ & $-\frac{d^{2}}{d \xi^{2}}\left(\frac{u_{b}}{L}\right)$ \\
\hline 1,3 & $\left(\frac{\lambda \bar{M}_{x p}}{L G D_{x x}}\right) \frac{d^{2} \theta_{z b}}{d \xi^{2}}+\lambda\left(\frac{\bar{V}_{y p}}{G D_{x x}}\right) \frac{d \theta_{z b}}{d \xi}$ & $\begin{array}{c}\lambda \bar{M}_{x p} / L G D_{x x}=5.4 \times 10^{-4} / L \\
\lambda \bar{V}_{y p} / G D_{x x}=5.8 \times 10^{-4} \\
\text { (negligible terms) }\end{array}$ & 0 \\
\hline 3,3 & $-\left[\frac{\left(J+D_{h h}\right)}{D_{x x} L^{2}}+\lambda \bar{N}_{p} \frac{\left(I_{x x}+I_{y y}\right)}{G D_{x x} A L^{2}}\right]$ & $\begin{array}{c}\left(J+D_{h h}\right) / D_{x x} L^{2} \\
=1.6 \times 10^{-2} / L^{2} \\
\lambda \bar{N}_{p}\left(I_{x x}+I_{y y}\right) / G D_{x x} A L^{2} \\
=3.7 \times 10^{-5} / L^{2} \\
<<\left(J+D_{h h}\right) / D_{x x} L^{2}\end{array}$ & {$\left[-\frac{\left(J+D_{h h}\right)}{D_{x x} L^{2}}\right] \frac{d^{2} \theta_{z b}}{d \xi^{2}}$} \\
\hline
\end{tabular}

* e.g., Entry $(1,3)$ denotes the third term in the first row in Eq. (A1)

The above approximations lead to the following simplified system of equilibrium equations 


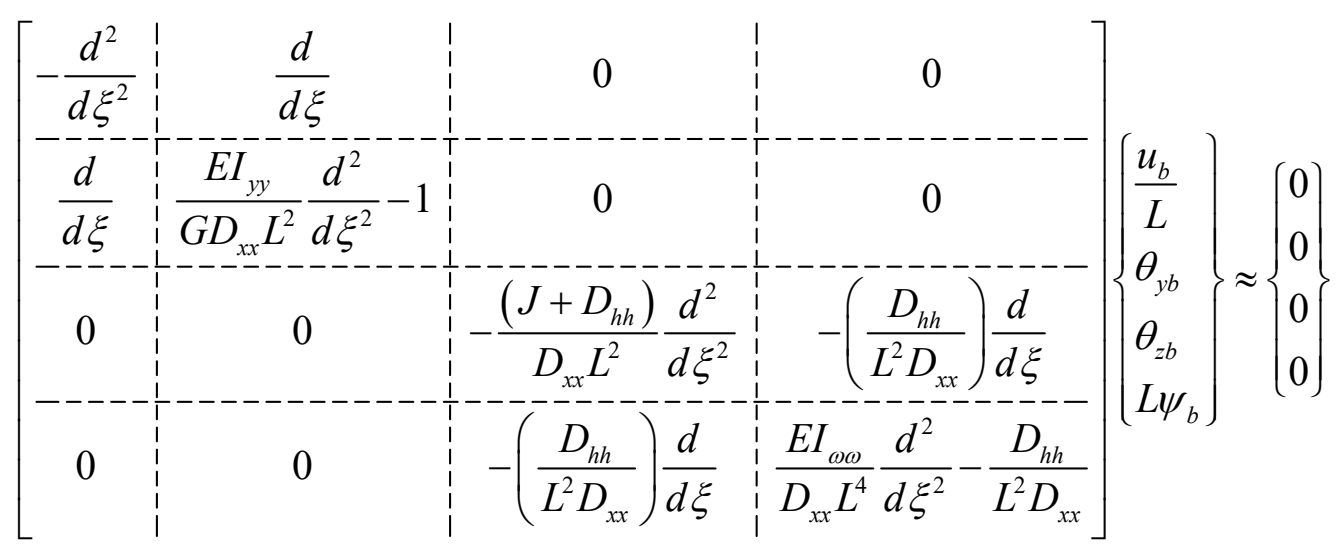

which are the non-dimensional form of Eqs. (10a-b) used to obtain the shape functions.

\section{Appendix B. Matrices needed to determine stiffness matrices}

This appendix provides explicit expressions for matrices stiffness components. In Eq. (20), the elastic stiffness matrix components $\left[K_{f}\right],\left[K_{s v}\right],\left[K_{s}\right]$ and geometric stiffness components $\left[K_{G}\right]_{N},\left[K_{G}\right]_{M},\left[K_{G}\right]_{V},\left[K_{G}\right]_{q y},\left[K_{G}\right]_{q z} \quad$ were expressed as functions of matrices $\left[M_{1}\right],\left[M_{2}\right], \ldots,\left[M_{8}\right]$. Matrices $\left[M_{1}\right],\left[M_{2}\right], \ldots,\left[M_{8}\right]$ are provided as follows:

\section{Elastic stiffness due to flexural stresses}

Matrix $\left[M_{1}\right]$ related to $\left[K_{f}\right]$ is given by

$$
\left[M_{1}\right]=E I_{y y}\left[\begin{array}{cc}
{\left[S_{1}\right]_{4 \times 4}} & {[0]_{4 \times 4}} \\
\hdashline[0]_{4 \times 4} & {\left[S_{2}\right]_{4 \times 4}}
\end{array}\right],\left[S_{1}\right]=\left[\begin{array}{cccc}
0 & 0 & 0 & 0 \\
& 0 & 0 & 0 \\
& & 4 L & 6 L^{2} \\
\text { Sym. } & & & 12 L^{3}
\end{array}\right],\left[S_{2}\right]=\left[\begin{array}{cccc}
0 & 0 & 0 & 0 \\
& 0 & 0 & 0 \\
& & \bar{a}_{3,3} & \bar{a}_{3,4} \\
\text { Sym. } & & & \bar{a}_{4,4}
\end{array}\right]
$$

$(\mathrm{B} 1 \mathrm{a}-\mathrm{c})$

in which, function $\bar{a}_{i, j}$ is defined as 
$\bar{a}_{i, j}=\frac{I_{\omega \omega} m_{i}^{2} m_{j}^{2}\left(e^{L\left(m_{i}+m_{j}\right)}-1\right)\left(D_{h h}+J\right)^{2}}{D_{h h}^{2}\left(m_{i}+m_{j}\right) I_{y y}}$

Elastic stiffness due to Saint-Venant shear stresses

Matrix $\left[M_{2}\right]$ related to $\left[K_{s v}\right]$ is given by

$\left[M_{2}\right]=G J\left[\begin{array}{c:c}{[0]_{4 \times 4}} & {[0]_{4 \times 4}} \\ \hdashline[0]_{4 \times 4} & {\left[S_{3}\right]_{4 \times 4}}\end{array}\right], \quad\left[S_{3}\right]=\left[\begin{array}{cccc}0 & 0 & 0 & 0 \\ & L & \bar{b}_{3} & \bar{b}_{4} \\ & & \bar{c}_{3,3} & \bar{c}_{3,4} \\ \text { Sym. } & & & \bar{c}_{4,4}\end{array}\right]$

in which, functions $\bar{b}_{i}$ and $\bar{c}_{i, j}$ are defined as

$\bar{b}_{i}=e^{L m_{i}}-1, \quad \bar{c}_{i, j}=\frac{m_{i} m_{j}\left(e^{L\left(m_{i}+m_{j}\right)}-1\right)}{m_{i}+m_{j}}$

Elastic stiffness due to other shear stresses

Matrix $\left[M_{3}\right]$ related to $\left[K_{s}\right]$ is given by

$\left[M_{3}\right]=\left[\begin{array}{c:c}{\left[S_{4}\right]_{4 \times 4}} & {[0]_{4 \times 4}} \\ \hdashline[0]_{4 \times 4} & {\left[S_{5}\right]_{4 \times 4}}\end{array}\right],\left[S_{4}\right]=\left[\begin{array}{cccc}0 & 0 & 0 & 0 \\ & 0 & 0 & 0 \\ & & 0 & 0 \\ \text { Sym. } & & & \bar{\alpha}\end{array}\right],\left[S_{5}\right]=\left[\begin{array}{cccc}0 & 0 & 0 & 0 \\ & 0 & 0 & 0 \\ & & \bar{d}_{3,3} & \bar{d}_{3,4} \\ \text { Sym. } & & & \bar{d}_{4,4}\end{array}\right]$

in which, function $\bar{d}_{i, j}$ and constant $\bar{\alpha}$ are defined as

$\bar{d}_{i, j}=\frac{G J^{2} m_{i} m_{j}\left(e^{L\left(m_{i}+m_{j}\right)}-1\right)}{D_{h h}\left(m_{i}+m_{j}\right)}, \quad \bar{\alpha}=\frac{36 E^{2} I_{y y}{ }^{2} L}{G D_{x x}}$ 


\section{Geometric stiffness due to normal forces}

Matrix $\left[M_{4}\right]$ related to $\left[K_{G}\right]_{N}$ is given by

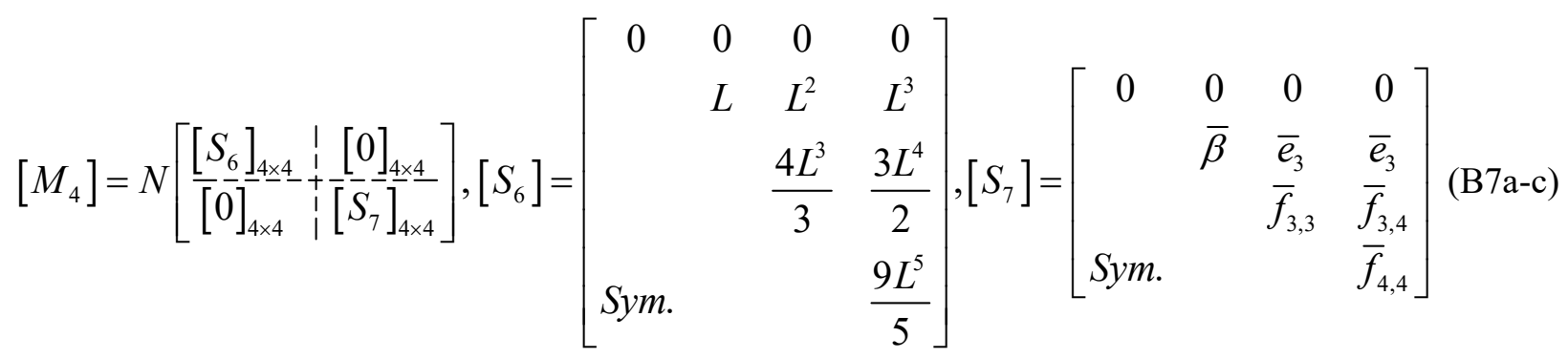

in which, functions $\bar{e}_{i}, \bar{f}_{i, j}$ and constant $\bar{\beta}$ are defined as

$\bar{e}_{i}=\frac{\left(e^{L m_{i}}-1\right)\left(I_{x x}+I_{y y}\right)}{A}, \quad \bar{f}_{i, j}=\frac{m_{i} m_{j}\left(e^{L\left(m_{i}+m_{j}\right)}-1\right)\left(I_{x x}+I_{y y}\right)}{A\left(m_{i}+m_{j}\right)}, \quad \bar{\beta}=\frac{L\left(I_{x x}+I_{y y}\right)}{A}$

\section{Geometric stiffness due to bending moments}

Matrix $\left[M_{5}\right]$ related to $\left[K_{G}\right]_{M}$ is given by

$\left[M_{5}\right]=M\left[\begin{array}{cc}{[0]_{4 \times 4}} & {\left[S_{8}\right]_{4 \times 4}} \\ \hdashline\left[S_{8}\right]_{4 \times 4}^{T} & {[0]_{4 \times 4}}\end{array}\right], \quad\left[S_{8}\right]=\left[\begin{array}{cccc}0 & 0 & 0 & 0 \\ 0 & 0 & 0 & 0 \\ 2 L & L^{2} & \bar{g}_{3} & \bar{g}_{4} \\ 3 L^{2} & \left(2 L^{3}+\frac{6 E I_{y y} L}{G D_{x x}}\right) & \bar{h}_{3} & \bar{h}_{4}\end{array}\right]$

in which, functions $\bar{g}_{i}$ and $\bar{h}_{i}$ are defined as

$\bar{g}_{i}=\frac{2\left(e^{L m_{i}}-1\right)}{m_{i}}, \quad \bar{h}_{i}=6\left(\frac{1}{m_{i}^{2}}+\frac{e^{L m_{i}}\left(L m_{i}-1\right)}{m_{i}^{2}}\right)+\frac{6 E I_{y y}\left(e^{L m_{i}}-1\right)}{G D_{x x}}$ 


\section{Geometric stiffness due to shear forces}

Matrix $\left[M_{6}\right]$ related to $\left[K_{G}\right]_{V}$ is given by

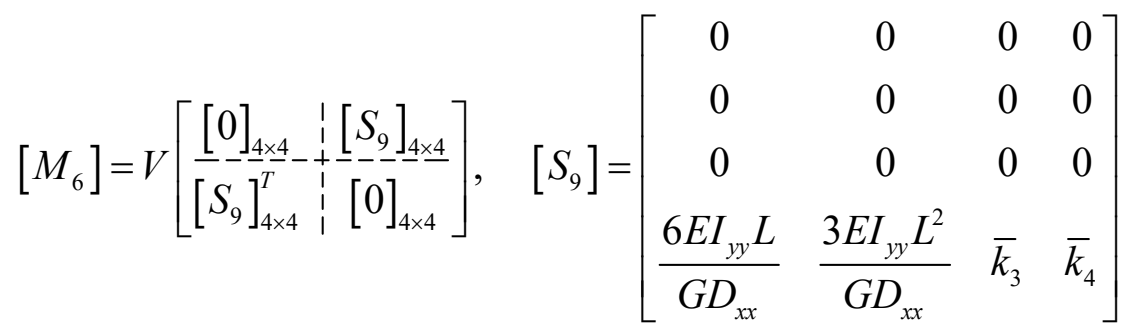

in which, function $\bar{k}_{i}$ is defined as

$$
\bar{k}_{i}=-\frac{6 E I_{y y}\left(e^{L m_{i}}-1\right)}{G D_{x x} m_{i}}
$$

\section{Geometric stiffness due to distributed transverse load}

Matrix $\left[M_{7}\right]$ related to $\left[K_{G}\right]_{q y}$ is given by

$$
\left[M_{7}\right]=q_{y} y_{q y}\left[\begin{array}{lll}
{[0]_{4 \times 4}} & {[0]_{4 \times 4}} \\
\hdashline[0]_{4 \times 4} & {\left[S_{10}\right]_{4 \times 4}}
\end{array}\right], \quad\left[S_{10}\right]=\left[\begin{array}{cccc}
L & \frac{L^{2}}{2} & \bar{l}_{3} & \bar{l}_{4} \\
& \frac{L^{3}}{3} & \bar{n}_{3} & \bar{n}_{4} \\
& & \bar{o}_{3,3} & \bar{o}_{3,4} \\
& & & \\
\text { Sym. } & & & \bar{o}_{4,4}
\end{array}\right]
$$

in which, functions $\bar{l}_{i}, \bar{n}_{i}$ and $\bar{o}_{i, j}$ are defined as

$$
\bar{l}_{i}=\frac{e^{L m_{i}}-1}{m_{i}}, \quad \bar{n}_{i}=\frac{1}{m_{i}^{2}}+\frac{e^{L m_{i}}\left(L m_{i}-1\right)}{m_{i}^{2}}, \quad \bar{o}_{i, j}=\frac{e^{L\left(m_{i}+m_{j}\right)}-1}{m_{i}+m_{j}}
$$




\section{Geometric stiffness due to distributed axial load}

Matrix $\left[M_{8}\right]$ related to $\left[K_{G}\right]_{q z}$ is given by

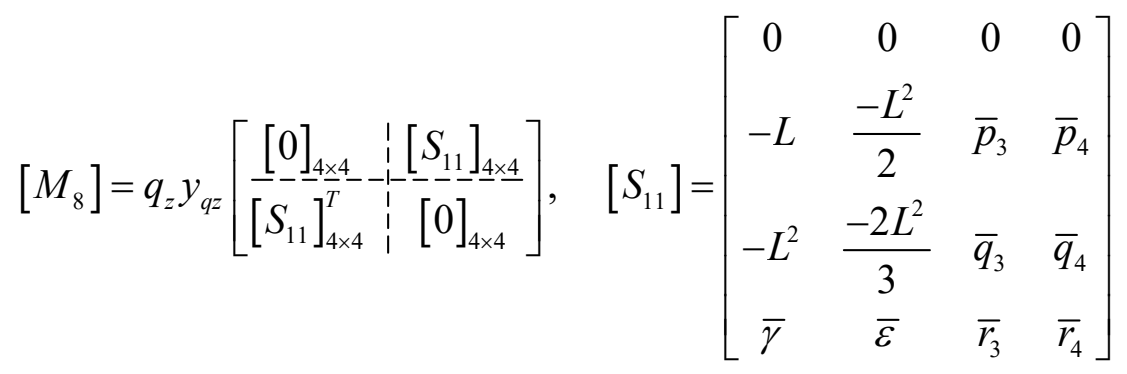

in which, functions $\bar{p}_{i}, \bar{q}_{i}, \bar{r}_{i}$ and constants $\bar{\gamma}$ and $\bar{\varepsilon}$ are defined as

$$
\begin{aligned}
& \bar{p}_{i}=\frac{\left(e^{L m_{i}}-1\right)}{m_{i}}, \quad \bar{q}_{i}=-2\left(\frac{1}{m_{i}^{2}}+\frac{e^{L m_{i}}\left(L m_{i}-1\right)}{m_{i}^{2}}\right), \quad \bar{\gamma}=\frac{-L\left(G D_{x x} L^{2}+6 E I_{y y}\right)}{G D_{x x}} \\
& \bar{r}_{i}=3\left(\frac{2}{m_{i}^{3}}-\frac{e^{L m_{i}}\left(L^{2} m_{i}^{2}-2 L m_{i}+2\right)}{m_{i}^{3}}\right)-\frac{6 E I_{y y}\left(e^{L m_{i}}-1\right)}{G D_{x x} m_{i}}, \quad \bar{\varepsilon}=\frac{-3 L^{2}\left(G D_{x x} L^{2}+4 E I_{y y}\right)}{4 G D_{x x}}
\end{aligned}
$$

\section{Load position matrix for concentrated transverse load}

When a member is subject to a concentrated transverse load $Q_{y}$ applied at $z=z_{Q y}$ and position $y_{Q y}$ relative to the shear center, the load function in Eq. (2g) can be expressed as $q_{y}(z)=Q_{y} \operatorname{Dirac}\left(z-z_{Q y}\right)$. Substituting this load function into Eq. (2g), one obtains a new geometric stiffness matrix $\left[K_{G}\right]_{Q y}$ due to load position effect relative to the shear center $S C$. Matrix $\left[M_{9}\right]$ related to $\left[K_{G}\right]_{Q y}$ is given by

$$
\left[M_{9}\right]=q_{y} y_{q y}\left[\begin{array}{c:c}
{[0]_{4 \times 4}} & {[0]_{4 \times 4}} \\
\hdashline[0]_{4 \times 4} & {\left[S_{12}\right]_{4 \times 4}}
\end{array}\right], \quad\left[S_{12}\right]=\left[\begin{array}{cccc}
1 & z_{Q y} & \overline{s_{3}} & \bar{s}_{4} \\
& z_{Q y}^{2} & \overline{t_{3}} & \overline{t_{4}} \\
& & \bar{u}_{3,3} & \bar{u}_{3,4} \\
& & & \bar{u}_{4,4}
\end{array}\right]
$$


in which, functions $\bar{s}_{i}, \bar{t}_{i}$ and $\bar{u}_{i, j}$ are defined as

$$
\overline{s_{i}}=e^{m_{i} z_{Q y}}, \quad \bar{t}_{i}=z_{Q y} e^{m_{i} z_{Q y}}, \quad \bar{u}_{i, j}=e^{z_{Q y}\left(m_{i}+m_{j}\right)}
$$

\section{Notation}

\begin{tabular}{|c|c|c|}
\hline$A$ & $=$ & Cross-sectional area \\
\hline$[B(z)]$ & $=$ & Matrix relating displacement fields to integration constants \\
\hline$\left\{C_{s t}\right\}$ & $=$ & Vector of integration constants \\
\hline$D_{h h}, D_{x x}$ & $=$ & Properties of cross-section related to shear deformation \\
\hline$d$ & $=$ & Depth of cross-section \\
\hline$\langle d(z)\rangle^{T}$ & $=$ & Field displacements \\
\hline E & $=$ & Modulus of elasticity \\
\hline$\{F\}$ & $=$ & Vector of Lagrange multipliers \\
\hline$G$ & $=$ & Shear modulus \\
\hline$[H]$ & $=$ & Matrix relating nodal displacements to integration constants \\
\hline$I_{x x}, I_{y y}$ & $=$ & $\begin{array}{l}\text { Moments of inertia of the cross-section about } \mathrm{x} \text {-axis and } \mathrm{y} \text {-axis } \\
\text { respectively }\end{array}$ \\
\hline$I_{\omega \omega}$ & $=$ & Warping Constant \\
\hline$J$ & $=$ & St. Venant torsional constant \\
\hline$\left[K_{f}\right]$ & $=$ & Stiffness matrix due to flexural stresses \\
\hline$\left[K_{G}\right]_{N}$ & $=$ & Geometric matrix due to normal forces \\
\hline$\left[K_{G}\right]_{M}$ & $=$ & Geometric matrix due to bending moments \\
\hline$\left[K_{G}\right]_{V}$ & $=$ & Geometric matrix due to shear forces \\
\hline$\left[K_{G}\right]_{q y}$ & $=$ & $\begin{array}{l}\text { Geometric matrix due to load position effect of the distributed } \\
\text { transverse load }\end{array}$ \\
\hline$\left[K_{G}\right]_{q z}$ & $=$ & $\begin{array}{l}\text { Geometric matrix due to load position effect of the distributed } \\
\text { axial load }\end{array}$ \\
\hline$\left[K_{G}\right]_{Q y}$ & $=$ & $\begin{array}{l}\text { Geometric matrix due to load position effect of the concentrated } \\
\text { transverse load }\end{array}$ \\
\hline$\left[K_{s}\right]$ & $=$ & Stiffness matrix due to other shear stresses \\
\hline$\left[K_{s v}\right]$ & $=$ & Stiffness matrix due to Saint Venant shear stresses \\
\hline$L$ & $=$ & Length of a finite element \\
\hline$L_{m}$ & $=$ & Span of the member \\
\hline
\end{tabular}




\begin{tabular}{|c|c|c|}
\hline$[L(z)]$ & $=$ & Matrix of shape functions \\
\hline$m_{i}$ & $=$ & Roots of quadratic eigenvalue problem \\
\hline$M_{1}, M_{2}$ & $=$ & Internal bending moment at both end of an element \\
\hline$M_{x p}(z)$ & $=$ & $\begin{array}{l}\text { Strong axis bending moment as obtained from pre-buckling } \\
\text { analysis }\end{array}$ \\
\hline$N_{1}, N_{2}$ & $=$ & Internal normal forces at both ends of an element \\
\hline$N_{p}(z)$ & $=$ & $\begin{array}{l}\text { Resultant of the normal stresses obtained from pre-buckling } \\
\text { Analysis }\end{array}$ \\
\hline$[P]$ & $=$ & $\begin{array}{l}\text { Matrix of user-input coefficients which linearly relate any set of } \\
\text { nodal displacements }\end{array}$ \\
\hline$q_{y}, q_{z}$ & $=$ & $\begin{array}{l}\text { Distributed load applied to a member acting along the } y-\text { and } z- \\
\text { direction respectively }\end{array}$ \\
\hline$[S]$ & $=$ & Structure elastic stiffness matrix \\
\hline$\left[S_{G}\right]$ & $=$ & Structure geometric stiffness matrix \\
\hline$\left[S_{i}\right](i=1$ to 12$)$ & $=$ & Sub-matrices needed to determine element stiffness matrices \\
\hline$u_{b}$ & $=$ & Lateral buckling displacement \\
\hline$\left\langle u_{N}\right\rangle^{T}$ & $=$ & Vector of nodal displacements \\
\hline$\left\{u_{s}\right\}$ & $=$ & Vector of unknown displacements of the structure \\
\hline$U$ & $=$ & Internal strain energy \\
\hline$V$ & $=$ & Load potential energy \\
\hline$V_{1}, V_{2}$ & $=$ & Internal shearing forces at both end of an element \\
\hline$V_{y p}(z)$ & $=$ & $\begin{array}{l}\text { Resultant of shear force component along y-direction obtained } \\
\text { from pre-buckling analysis }\end{array}$ \\
\hline$x, y, z$ & $=$ & Cartesian coordinates \\
\hline$\beta$ & $=$ & End moment ratio \\
\hline$\lambda$ & $=$ & Load multiplier \\
\hline$\pi$ & $=$ & Total potential energy \\
\hline$\theta_{y b}, \theta_{z b}$ & $=$ & Buckling rotation angles about $\mathrm{y}, \mathrm{z}$ axes, respectively \\
\hline$\omega(s)$ & $=$ & Warping function \\
\hline$\psi_{b}$ & $=$ & Warping deformation (1/Length) \\
\hline
\end{tabular}

\section{References}

[1] L. Wu, M. Mohareb, Buckling of shear deformable thin-walled members-I. Variational principle and analytical solutions, Thin Wall Struct., 49 (2011a) 197-207. 
[2] L. Wu, M. Mohareb, Buckling formulation for shear deformable thin-walled members-II. Finite element formulation, Thin Wall Struct., 49 (2011b) 208-222.

[3] M.G. Salvadori, Lateral Buckling of I-Beams, Trans. of the Amer. Soc. of Civil Eng., 120 (1955) 1165-1177.

[4] S. Poley, Lateral Buckling of Cantilevered I-Beams Under Uniform Load, Trans. of the Amer. Soc. of Civil Eng., 121 (1956) 786-790.

[5] W.J. Austin, S. Yegian, T.P. Tung, Lateral Buckling of Elastically End-Restrained I-Beams, Trans. of the Amer. Soc. of Civil Eng., 122 (1957) 374-388.

[6] A.J. Hartmann, Elastic Lateral Buckling of Continuous Beams, J. of the Struct. Div., 93 (1967) 11-26.

[7] D. Krajcinovic, A consistent discrete elements technique for thinwalled assemblages, Int. J. of Solids and Struct., 5 (1969) 639-662.

[8] R.S. Barsoum, R.H. Gallagher, Finite element analysis of torsional and torsional-flexural stability problems, International Journal for Numerical Methods in Engineering, 2 (1970) 335352.

[9] G. Powell, R. Klingner, Elastic Lateral Buckling of Steel Beams, J. of the Struct. Div., 96 (1970) 1919-1932.

[10] R.H. Gallagher, J. Padlog, DISCRETE ELEMENT APPROACH TO STRUCTURAL INSTABILITY ANALYSIS, AIAA Journal, 1 (1963) 1437-1439.

[11] D.A. Nethercot, K.C. Rockey, Finite element solutions for the buckling of columns and beams, Int. J. of Mech. Sci., 13 (1971) 945-949.

[12] D.A. Nethercot, The Effective Length of Cantilevers as Governed by Lateral Buckling, The Struct. Eng., 51 (1973a) 161-168.

[13] D.A. Nethercot, buckling of laterally or torsionally restrained beams, J. of the Eng. Mech. Div., ASCE, 99 (1973b) 773-791.

[14] S. Kitipornchai, N.J. Richter, Elastic lateral buckling of I-beams with discrete intermediate restraints, Civil Eng. Trans., Inst. of Eng., Australia, CE20 (1978) 105-111.

[15] S. Kitipornchai, P. Dux, N. Richter, Buckling and Bracing of Cantilevers, J. of Struct. Eng., 110 (1984) 2250-2262.

[16] M. Assadi, C. Roeder, Stability of Continuously Restrained Cantilevers, J. of Eng. Mech., 111 (1985) 1440-1456.

[17] T. Geng-Shu, C. Shao-Fan, Buckling of laterally and torsionally braced beams, J. of Constr. Steel Res., 11 (1988) 41-55.

[18] Y.C. Wang, D.A. Nethercot, Bracing requirements for laterally unrestrained beams, J. of Constr. Steel Res., 17 (1990) 305-315.

[19] M.M. Attard, General non-dimensional equation for lateral buckling, Thin Wall Struct., 9 (1990) 417-435.

[20] C. Albert, H.S. Essa, D.J.L. Kennedy, Distortional buckling of steel beams in cantileversuspended span construction, Can. J. of Civil Eng., 19 (1992) 767-780.

[21] H. Essa, D. Kennedy, Design of Cantilever Steel Beams: Refined Approach, J. of Struct. Eng., 120 (1994) 2623-2636.

[22] H. Essa, D. Kennedy, Design of Steel Beams in Cantilever-Suspended-Span Construction, J. of Struct. Eng., 121 (1995) 1667-1673.

[23] C.M. Wang, K.K. Ang, L. Wang, Optimization of bracing and internal support locations for beams against lateral buckling, Struct. Opt., 9 (1995) 12-17. 
[24] H.U. Akay, C.P. Johnson, K.M. Will, Lateral and Local Buckling of Beams and Frames, J. of the Struct. Div., ASCE, ST9, 103 (1977) 1821-1832.

[25] K.M. Choo, Building Program BASP for Use on a Micro Computer in, University of Texas at Austin, 1987.

[26] J. Yura, Fundamentals of beam bracing, Eng. J., AISC, (First Quarter) (2001) 11-26.

[27] N.-H. Lim, N.-H. Park, Y.-J. Kang, I.-H. Sung, Elastic buckling of I-beams under linear moment gradient, Int. J. of Solids and Struct., 40 (2003) 5635-5647.

[28] J.S. Park, J.M. Stallings, Y.J. Kang, Lateral-torsional buckling of prismatic beams with continuous top-flange bracing, J. of Constr. Steel Res., 60 (2004) 147-160.

[29] K.M. Özdemir, C. Topkaya, Lateral buckling of overhanging crane trolley monorails, Eng. Struct., 28 (2006) 1162-1172.

[30] M.A. Serna, A. López, I. Puente, D.J. Yong, Equivalent uniform moment factors for lateraltorsional buckling of steel members, J. of Constr. Steel Res., 62 (2006) 566-580.

[31] A. Andrade, D. Camotim, P. Providência e Costa, On the evaluation of elastic critical moments in doubly and singly symmetric I-section cantilevers, J. of Constr. Steel Res., 63 (2007) 894-908.

[32] Eurocode3, Comité Européen de Normalisation (CEN), Design of Steel Structures, in: Part 1-1: General rules and rules for buildings (ENV 1993-1-1), Brussels, 1992.

[33] B. Larue, A. Khelil, M. Gueury, Elastic flexural-torsional buckling of steel beams with rigid and continuous lateral restraints, J. of Constr. Steel Res., 63 (2007) 692-708.

[34] A. Khelil, B. Larue, Simple solutions for the flexural-torsional buckling of laterally restrained I-beams, Eng. Struct., 30 (2008) 2923-2934.

[35] N.S. Trahair, Lateral buckling of monorail beams, Eng. Struct., 30 (2008) 3213-3218.

[36] C.T. Nguyen, J. Moon, V.N. Le, H.-E. Lee, Lateral-torsional buckling of I-girders with discrete torsional bracings, J. of Constr. Steel Res., 66 (2010) 170-177.

[37] R.E. Erkmen, M. Mohareb, Buckling analysis of thin-walled open members - A finite element formulation, Thin Wall Struct., 46 (2008b) 618-636.

[38] R.E. Erkmen, M. Mohareb, Buckling analysis of thin-walled open members-A complementary energy variational principle, Thin Wall Struct., 46 (2008a) 602-617.

[39] R.E. Erkmen, M. Mohareb, M. Bradford, Complementary Energy Based Formulation for Torsional Buckling of Columns, J. of Eng. Mech., 135 (2009) 1420-1426.

[40] M.M. Attard, M.-Y. Kim, Lateral buckling of beams with shear deformations - A hyperelastic formulation, Int. J. of Solids and Struct., 47 (2010) 2825-2840.

[41] R.E. Erkmen, M.M. Attard, Lateral-torsional buckling analysis of thin-walled beams including shear and pre-buckling deformation effects, Int. J. of Mech. Sci., 53 (2011) 918-925.

[42] R.E. Erkmen, Shear deformable hybrid finite-element formulation for buckling analysis of thin-walled members, Finite Elements in Analysis and Design, 82 (2014) 32-45.

[43] N.-I. Kim, J. Lee, Lateral buckling of shear deformable laminated composite I-beams using the finite element method, Int. J. of Mech. Sci., 68 (2013) 246-257.

[44] N.-I. Kim, D.-H. Choi, Super convergent shear deformable finite elements for stability analysis of composite beams, Composites Part B: Engineering, 44 (2013) 100-111.

[45] H.A.F.A. Santos, Complementary-Energy Methods for Geometrically Non-linear Structural Models: An Overview and Recent Developments in the Analysis of Frames, Arch Computat Methods Eng, 18 (2011) 405-440. 
[46] H.A.F.A. Santos, Variationally consistent force-based finite element method for the geometrically non-linear analysis of Euler-Bernoulli framed structures, Finite Elements in Analysis and Design, 53 (2012) 24-36.

[47] N.S. Trahair, Flexural-torsional buckling of structures, USA: CRC Press, 1993.

[48] A. Sahraei, L. Wu, M. Mohareb, Finite element formulation for lateral torsional buckling analysis of shear deformable mono-symmetric thin-walled members, Thin Wall Struct., 89 (2015) 212-226.

[49] M. Hjaji, M. Mohareb, Finite-Element Formulation for the Linear Steady-State Response of Asymmetric Thin-Walled Members under Harmonic Forces, J. of Eng. Mech., (2014) 04014126. [50] A.I.S.C. American Institute of Steel Construction, Specification for Structural Steel Buildings, in: ANSI/AISC 360-10, Chicago, IL, 2010. 